\title{
STATIONARITY AND ERGODICITY FOR AN AFFINE TWO-FACTOR MODEL
}

\author{
MÁTYÁS BARCZY, * University of Debrecen \\ LEIF DÖRING, ${ }^{* *}$ Universität Zürich \\ ZENGHU LI, ${ }^{* * *}$ Beijing Normal University \\ GYULA PAP, ${ }^{* * * *}$ University of Szeged
}

\begin{abstract}
We study the existence of a unique stationary distribution and ergodicity for a twodimensional affine process. Its first coordinate process is supposed to be a so-called $\alpha$-root process with $\alpha \in(1,2]$. We prove the existence of a unique stationary distribution for the affine process in the $\alpha \in(1,2]$ case; furthermore, we show ergodicity in the $\alpha=2$ case.
\end{abstract}

Keywords: Affine process; stationary distribution; ergodicity; Foster-Lyapunov criteria

2010 Mathematics Subject Classification: Primary 60J25

Secondary $37 \mathrm{~A} 25$

\section{Introduction}

We consider the two-dimensional affine process (affine two-factor model)

$$
\begin{aligned}
\mathrm{d} Y_{t}=\left(a-b Y_{t}\right) \mathrm{d} t+\sqrt[\alpha]{Y_{t-}} \mathrm{d} L_{t}, & t \geq 0, \\
\mathrm{~d} X_{t}=\left(m-\theta X_{t}\right) \mathrm{d} t+\sqrt{Y_{t}} \mathrm{~d} B_{t}, & t \geq 0,
\end{aligned}
$$

where $a>0, b, \theta, m \in \mathbb{R}, \alpha \in(1,2],\left(L_{t}\right)_{t \geq 0}$ is a spectrally positive $\alpha$-stable Lévy process with Lévy measure $C_{\alpha} z^{-1-\alpha} \mathbf{1}_{\{z>0\}}$, with $C_{\alpha}:=(\alpha \Gamma(-\alpha))^{-1}$ (where $\Gamma$ denotes the gamma function) in the $\alpha \in(1,2)$ case and a standard Wiener process in the $\alpha=2$ case, and $\left(B_{t}\right)_{t \geq 0}$ is an independent standard Wiener process. Owing to the almost-sure continuity of the sample paths of a standard Wiener process, instead of $\sqrt{Y_{t-}}$ we can write $\sqrt{Y_{t}}$ in the first stochastic differential equation (SDE) of (1.1), with $Y$ the so-called Cox-Ingersol-Ross (CIR) process in the $\alpha=2$ case and in the $\alpha \in(1,2)$ case, the $\alpha$-root process. Note also that the process $\left(Y_{t}\right)_{t \geq 0}$ given by the first SDE of (1.1) is a continuous-state branching process with immigration, with branching mechanism $b z+z^{\alpha} / \alpha, z \geq 0$, and immigration mechanism $a z, z \geq 0$ (for more details, see the proof of Theorem 3.1(i)). Chen and Joslin [8] found several applications of model (1.1) with $\alpha=2$ in financial mathematics; see their Equations (25) and (26).

The process $(Y, X)$ given by (1.1) is a special affine process. The set of affine processes contains a large class of important Markov processes, such as continuous-state branching processes and Orstein-Uhlenbeck processes. Furthermore, a lot of models in financial mathematics

Received 13 February 2013; revision received 19 September 2013.

* Postal address: Faculty of Informatics, University of Debrecen, Pf. 12, H-4010 Debrecen, Hungary.

Email address: barczy.matyas@inf.unideb.hu

** Postal address: Institut für Mathematik, Universität Zürich, Winterthurerstrasse 190, CH-8057 Zürich, Switzerland.

*** Postal address: School of Mathematical Sciences, Beijing Normal University, Beijing, 100875, P. R. China.

**** Postal address: Bolyai Institute, University of Szeged, Aradi vértanúk tere 1, H-6720 Szeged, Hungary. 
are affine, such as the Heston model [18], the model of Barndorff-Nielsen and Shephard [4], or the model due to Carr and $\mathrm{Wu}$ [7]. A precise mathematical formulation and a complete characterization of regular affine processes are due to Duffie et al. [13]. Later several authors contributed to the theory of general affine processes: to name a few, Andersen and Piterbarg [1], Dawson and Li [12], Filipović and Mayerhofer [14], Glasserman and Kim [17], Jena et al. [21], and Keller-Ressel et al. [24].

In this work we concentrate on the study of the existence of a unique stationary distribution and ergodicity of the affine process given by SDE (1.1). These kinds of result are important in their own right and they can be used to study parameter estimation for the given model. For the existing results on ergodicity of affine processes, see the beginning of Section 3.

Next we give a brief overview of the structure of the paper. Section 2 is devoted to a preliminary discussion of the existence and uniqueness of a strong solution of SDE (1.1) by proving that this solution is indeed an affine process; see Theorem 2.1. In Section 3 we prove the existence of a unique stationary distribution for the affine process given by (1.1) in both the $\alpha \in(1,2)$ and $\alpha=2$ cases, provided that $a>0, b>0$, and $\theta>0$; see Theorem 3.1. In Section 4 , for the $\alpha=2$ case, we prove ergodicity of the process in question provided that $a>0, b>0$, and $\theta>0$, and we also show that the unique stationary distribution of the process is absolutely continuous and has finite (mixed) moments of any order by calculating some moments explicitly; see Theorems 4.1 and 4.2, respectively.

In a forthcoming paper we will use our results to study parameter estimation for the process given by SDE (1.1).

\section{The affine two-factor model}

Let $\mathbb{N}, \mathbb{Z}_{+}, \mathbb{R}$, and $\mathbb{R}_{+}$denote the sets of positive integers, nonnegative integers, real numbers, and nonnegative real numbers, respectively. By $\|x\|$ and $\|A\|$ we denote the Euclidean norm of a vector $x \in \mathbb{R}^{m}$ and the induced matrix norm $\|A\|=\sup \left\{\|A x\|: x \in \mathbb{R}^{m},\|x\|=1\right\}$ of a matrix $A \in \mathbb{R}^{n \times m}$, respectively. By $\mathcal{C}^{2}\left(\mathbb{R}_{+} \times \mathbb{R}, \mathbb{R}\right), \mathcal{C}_{c}^{2}\left(\mathbb{R}_{+} \times \mathbb{R}, \mathbb{R}\right)$, and $\mathcal{C}_{c}^{\infty}\left(\mathbb{R}_{+} \times \mathbb{R}, \mathbb{R}\right)$, we denote the set of twice continuously differentiable real-valued functions on $\mathbb{R}_{+} \times \mathbb{R}$, the set of twice continuously differentiable real-valued functions on $\mathbb{R}_{+} \times \mathbb{R}$ with compact support, and the set of infinitely differentiable real-valued functions on $\mathbb{R}_{+} \times \mathbb{R}$ with compact support, respectively. Convergence in distribution will be denoted by ' $\stackrel{\mathrm{D}}{\rightarrow}$ '.

Let $\left(\Omega, \mathcal{F},\left(\mathscr{F}_{t}\right)_{t \geq 0}, \mathbb{P}\right)$ be a filtered probability space satisfying the usual conditions, i.e. $(\Omega, \mathcal{F}, \mathbb{P})$ is complete, the filtration $\left(\mathcal{F}_{t}\right)_{t \geq 0}$ is right continuous, and $\mathcal{F}_{0}$ contains all the $\mathbb{P}$-null sets in $\mathcal{F}$. Let $\left(B_{t}\right)_{t \geq 0}$ be a standard $\left(\mathcal{F}_{t}\right)_{t \geq 0}$-Wiener process, and let $\left(L_{t}\right)_{t \geq 0}$ be a spectrally positive, $\left(\mathscr{F}_{t}\right)_{t \geq 0}$-stable process with index $\alpha \in(1,2]$. We assume that $B$ and $L$ are independent. If $\alpha=2$, we understand that $L$ is a standard $\left(\mathcal{F}_{t}\right)_{t \geq 0}$-Wiener process. If $\alpha \in(1,2)$, we understand that $L$ is a $\left(\mathscr{F}_{t}\right)_{t \geq 0}$-Lévy process with Lévy-Khintchine formula

$$
\mathbb{E}\left(\mathrm{e}^{\mathrm{i} u L_{1}}\right)=\exp \left\{\int_{0}^{\infty}\left(\mathrm{e}^{\mathrm{i} u z}-1-\mathrm{i} u z\right) C_{\alpha} z^{-1-\alpha} \mathrm{d} z\right\}, \quad u \in \mathbb{R},
$$

where $C_{\alpha}=(\alpha \Gamma(-\alpha))^{-1}$. Recall that in the $\alpha \in(1,2)$ case the Lévy-Itô representation of $L$ takes the form

$$
L_{t}=\int_{(0, t]} \int_{(0, \infty)} z \tilde{N}(\mathrm{~d} s, \mathrm{~d} z), \quad t \geq 0,
$$

where $\widetilde{N}(\mathrm{~d} s, \mathrm{~d} z)$ is a compensated Poisson random measure on $(0, \infty)^{2}$ with intensity measure $C_{\alpha} z^{-1-\alpha} \mathbf{1}_{\{z>0\}} \mathrm{d} s \mathrm{~d} z$. 
We note that in Remark 2.1 of [3] we shed some light on the definition of the stochastic integral with respect to the spectrally positive $\alpha$-stable process $L$ in the first SDE of (1.1) in the $\alpha \in(1,2)$ case.

Theorem 2.1. Let $\left(\eta_{0}, \zeta_{0}\right)$ be a random vector independent of $\left(L_{t}, B_{t}\right)_{t \geq 0}$, satisfying $\mathbb{P}\left(\eta_{0} \geq\right.$ $0)=1$. Then, for all $a>0, b, m, \theta \in \mathbb{R}$ and $\alpha \in(1,2]$, there is a (pathwise) unique strong solution $\left(Y_{t}, X_{t}\right)_{t \geq 0}$ of $\operatorname{SDE}(1.1)$ such that $\mathbb{P}\left(\left(Y_{0}, X_{0}\right)=\left(\eta_{0}, \zeta_{0}\right)\right)=1$ and $\mathbb{P}\left(Y_{t} \geq\right.$ 0 for all $t \geq 0)=1$. Furthermore, we have

$$
Y_{t}=\mathrm{e}^{-b(t-s)}\left(Y_{s}+a \int_{s}^{t} \mathrm{e}^{-b(s-u)} \mathrm{d} u+\int_{s}^{t} \mathrm{e}^{-b(s-u)} \sqrt[\alpha]{Y_{u-}} \mathrm{d} L_{u}\right)
$$

for $0 \leq s \leq t$ and

$$
X_{t}=\mathrm{e}^{-\theta(t-s)}\left(X_{s}+m \int_{s}^{t} \mathrm{e}^{-\theta(s-u)} \mathrm{d} u+\int_{s}^{t} \mathrm{e}^{-\theta(s-u)} \sqrt{Y_{u}} \mathrm{~d} B_{u}\right)
$$

for $0 \leq s \leq t$. Moreover, $\left(Y_{t}, X_{t}\right)_{t \geq 0}$ is a regular affine process with infinitesimal generator

$$
\begin{aligned}
(\mathcal{A} f)(y, x)= & (a-b y) f_{1}^{\prime}(y, x)+(m-\theta x) f_{2}^{\prime}(y, x)+\frac{1}{2} y f_{2,2}^{\prime \prime}(y, x) \\
& +y \int_{0}^{\infty}\left(f(y+z, x)-f(y, x)-z f_{1}^{\prime}(y, x)\right) C_{\alpha} z^{-1-\alpha} \mathrm{d} z
\end{aligned}
$$

in the $\alpha \in(1,2)$ case, and

$$
(\mathcal{A} f)(y, x)=(a-b y) f_{1}^{\prime}(y, x)+(m-\theta x) f_{2}^{\prime}(y, x)+\frac{1}{2} y\left(f_{1,1}^{\prime \prime}(y, x)+f_{2,2}^{\prime \prime}(y, x)\right)
$$

in the $\alpha=2$ case, where $(y, x) \in \mathbb{R}_{+} \times \mathbb{R}, f \in \mathcal{C}_{c}^{2}\left(\mathbb{R}_{+} \times \mathbb{R}, \mathbb{R}\right)$, and $f_{i}^{\prime}, i=1,2$, and $f_{i, j}^{\prime \prime}, i, j \in\{1,2\}$, denote the first-and second-order partial derivatives of $f$ with respect to the $i$ th, and ith and $j$ th variables.

Proof. By Theorem 6.2 or Corollary 6.3 of [16] (for the $\alpha \in(1,2)$ case) and by the Yamada and Watanabe theorem (see, e.g. [22, Proposition 5.2.13]) and [19, Example 8.2, p. 221] (for the $\alpha=2$ case), there is a (pathwise) unique, nonnegative strong solution $\left(Y_{t}\right)_{t \geq 0}$ of the first equation in (1.1) with any initial value $\eta_{0}$ satisfying $\mathbb{P}\left(\eta_{0} \geq 0\right)=1$.

Using Itô's formula, we can check (2.1) and (2.2) together with pathwise uniqueness for (1.1) (for more details, see the proof of Theorem 2.2 of [3]).

We now check that $\left(Y_{t}, X_{t}\right)_{t \geq 0}$ is a regular affine process with the given infinitesimal generator. We may and do suppose that the initial value is deterministic, say, $\left(Y_{0}, X_{0}\right)=$ $\left(y_{0}, x_{0}\right) \in \mathbb{R}_{+} \times \mathbb{R}$, since the infinitesimal generator of a time-homogeneous Markov process does not depend on the initial value of the Markov process.

In the $\alpha=2$ case, by Itô's formula, for all $f \in \mathcal{C}_{c}^{2}\left(\mathbb{R}_{+} \times \mathbb{R}, \mathbb{R}\right)$, we have

$$
f\left(Y_{t}, X_{t}\right)=f\left(y_{0}, x_{0}\right)+\int_{0}^{t}(\mathcal{A} f)\left(Y_{s}, X_{s}\right) \mathrm{d} s+M_{t}(f), \quad t \geq 0,
$$

where

$$
M_{t}(f):=\int_{0}^{t} f_{1}^{\prime}\left(Y_{s}, X_{s}\right) \sqrt{Y_{s}} \mathrm{~d} L_{s}+\int_{0}^{t} f_{2}^{\prime}\left(Y_{s}, X_{s}\right) \sqrt{Y_{s}} \mathrm{~d} B_{s}, \quad t \geq 0
$$


and $\mathcal{A} f$ is given by (2.4). It remains to show that $\left(M_{t}(f)\right)_{t \geq 0}$ is a local martingale with respect to the filtration $\left(\mathcal{F}_{t}\right)_{t \geq 0}$. However, we prove that it is a square-integrable martingale with respect to the filtration $\left(\mathcal{F}_{t}\right)_{t \geq 0}$, since

$$
\begin{array}{ll}
\int_{0}^{t} \mathbb{E}\left(\left(f_{1}^{\prime}\left(Y_{s}, X_{s}\right)\right)^{2} Y_{s}\right) \mathrm{d} s \leq C_{1} \int_{0}^{t} \mathbb{E}\left(Y_{s}\right) \mathrm{d} s<\infty, & t \geq 0, \\
\int_{0}^{t} \mathbb{E}\left(\left(f_{2}^{\prime}\left(Y_{s}, X_{s}\right)\right)^{2} Y_{s}\right) \mathrm{d} s \leq C_{2} \int_{0}^{t} \mathbb{E}\left(Y_{s}\right) \mathrm{d} s<\infty, & t \geq 0,
\end{array}
$$

with some constants $C_{1}>0$ and $C_{2}>0$, where the finiteness of the integrals follows from $\mathbb{E}\left(Y_{s}\right)=\mathrm{e}^{-b s} y_{0}+a \int_{0}^{s} \mathrm{e}^{-b u} \mathrm{~d} u, s \geq 0$; see, e.g. [10, Equation (19)], [20, Theorem 6.3.3.1], or Proposition 3.2 of [2].

In the $\alpha \in(1,2)$ case, by the Lévy-Itô representation of $L$, we can rewrite SDE (1.1) in integral form:

$$
\begin{gathered}
Y_{t}=y_{0}+\int_{0}^{t}\left(a-b Y_{s}\right) \mathrm{d} s+\int_{0}^{t} \int_{0}^{\infty} z \sqrt[\alpha]{Y_{s-}} \tilde{N}(\mathrm{~d} s, \mathrm{~d} z), \quad t \geq 0, \\
X_{t}=x_{0}+\int_{0}^{t}\left(m-\theta X_{s}\right) \mathrm{d} s+\int_{0}^{t} \sqrt{Y_{s}} \mathrm{~d} B_{s}, \quad t \geq 0 .
\end{gathered}
$$

By Itô's formula, for all $f \in \mathcal{C}_{c}^{2}\left(\mathbb{R}_{+} \times \mathbb{R}, \mathbb{R}\right)$, we have

$$
f\left(Y_{t}, X_{t}\right)=f\left(y_{0}, x_{0}\right)+\int_{0}^{t}(\mathcal{A} f)\left(Y_{s}, X_{s}\right) \mathrm{d} s+M_{t}(f), \quad t \geq 0,
$$

where

$$
\begin{aligned}
M_{t}(f):= & \int_{0}^{t} f_{2}^{\prime}\left(Y_{s}, X_{s}\right) \sqrt{Y_{s}} \mathrm{~d} B_{s} \\
& +\int_{0}^{t} \int_{0}^{\infty}\left(f\left(Y_{s-}+z \sqrt[\alpha]{Y_{s-}}, X_{s-}\right)-f\left(Y_{s-}, X_{s-}\right)\right) \tilde{N}(\mathrm{~d} s, \mathrm{~d} z), \quad t \geq 0,
\end{aligned}
$$

and, by the change of variable $z \sqrt[\alpha]{y}:=\widetilde{z}$,

$$
\begin{aligned}
(\mathscr{A} f)(y, x):= & (a-b y) f_{1}^{\prime}(y, x)+(m-\theta x) f_{2}^{\prime}(y, x)+\frac{1}{2} y f_{2,2}^{\prime \prime}(y, x) \\
& +y \int_{0}^{\infty}\left(f(y+\widetilde{z}, x)-f(y, x)-\widetilde{z} f_{1}^{\prime}(y, x)\right) C_{\alpha} z^{-1-\alpha} \mathrm{d} \widetilde{z}
\end{aligned}
$$

for $(y, x) \in \mathbb{R}_{+} \times \mathbb{R}$ and $f \in \mathcal{C}_{c}^{2}\left(\mathbb{R}_{+} \times \mathbb{R}, \mathbb{R}\right)$. It remains to show that $\left(M_{t}(f)\right)_{t \geq 0}$ is a local martingale with respect to the filtration $\left(\mathcal{F}_{t}\right)_{t \geq 0}$. However, we prove that it is a martingale with respect to the filtration $\left(\mathcal{F}_{t}\right)_{t \geq 0}$. The martingale property of $\int_{0}^{t} f_{2}^{\prime}\left(Y_{s}, X_{s}\right) \sqrt{Y_{s}} \mathrm{~d} B_{s}, t \geq 0$, follows in the same way as in the $\alpha=2$ case using the fact that there exists some constant $C_{3}>0$ such that $\mathbb{E}\left(Y_{t}\right) \leq C_{3}\left(1+y_{0} \mathrm{e}^{-b t / \alpha}\right)$ for all $t \geq 0$; see, e.g. Formula (2.7) or Proposition 2.10 of [26]. Now we check that

$$
M_{t}^{1}(f):=\int_{0}^{t} \int_{0}^{\infty}\left(f\left(Y_{s-}+z \sqrt[\alpha]{Y_{s-}}, X_{s-}\right)-f\left(Y_{s-}, X_{s-}\right)\right) \tilde{N}(\mathrm{~d} s, \mathrm{~d} z), \quad t \geq 0,
$$

is a martingale. For all $n \in \mathbb{N}$, let

$$
\begin{aligned}
& M_{t}^{2, n}(f):=\int_{0}^{t} \int_{1}^{\infty}\left(f\left(\left(Y_{s-} \wedge n\right)+z \sqrt[\alpha]{Y_{s-} \wedge n}, X_{s-}\right)-f\left(Y_{s-} \wedge n, X_{s-}\right)\right) \tilde{N}(\mathrm{~d} s, \mathrm{~d} z) \\
& M_{t}^{3, n}(f):=\int_{0}^{t} \int_{0}^{1}\left(f\left(\left(Y_{s-} \wedge n\right)+z \sqrt[\alpha]{Y_{s-} \wedge n}, X_{s-}\right)-f\left(Y_{s-} \wedge n, X_{s-}\right)\right) \tilde{N}(\mathrm{~d} s, \mathrm{~d} z)
\end{aligned}
$$


By Taylor's theorem, we have

$$
\left|f\left(\left(Y_{s-} \wedge n\right)+z \sqrt[\alpha]{Y_{s-} \wedge n}, X_{s-}\right)-f\left(Y_{s-} \wedge n, X_{s-}\right)\right| \leq z \sqrt[\alpha]{Y_{s-} \wedge n} \sup _{(y, x) \in \mathbb{R}_{+} \times \mathbb{R}}\left|f_{1}^{\prime}(y, x)\right|
$$

for $z \in \mathbb{R}_{+}$. Since

$$
\begin{aligned}
& \mathbb{E}\left(\int_{0}^{t} \int_{1}^{\infty}\left|f\left(\left(Y_{s-} \wedge n\right)+z \sqrt[\alpha]{Y_{s-} \wedge n}, X_{s-}\right)-f\left(Y_{s-} \wedge n, X_{s-}\right)\right| C_{\alpha} z^{-1-\alpha} \mathrm{d} s \mathrm{~d} z\right) \\
& \quad \leq\left(\sup _{(y, x) \in \mathbb{R}_{+} \times \mathbb{R}}\left|f_{1}^{\prime}(y, x)\right|\right) \int_{0}^{t} \int_{1}^{\infty} \mathbb{E}\left(\sqrt[\alpha]{Y_{s} \wedge n}\right) C_{\alpha} z^{-\alpha} \mathrm{d} s \mathrm{~d} z \\
& \quad \leq C_{4} n^{1 / \alpha} t \int_{1}^{\infty} z^{-\alpha} \mathrm{d} z \\
& \quad=C_{4} n^{1 / \alpha} \frac{t}{\alpha-1} \\
& \quad<\infty, \quad t \in \mathbb{R}_{+},
\end{aligned}
$$

with some constant $C_{4}>0$, by Lemma 3.1 of [19, p. 62], $\left(M_{t}^{2, n}(f)\right)_{t \geq 0}$ is a martingale with respect to the filtration $\left(\mathcal{F}_{t}\right)_{t \geq 0}$. Furthermore, since

$$
\begin{aligned}
& \mathbb{E}\left(\int_{0}^{t} \int_{0}^{1}\left(f\left(\left(Y_{s-} \wedge n\right)+z \sqrt[\alpha]{Y_{s-} \wedge n}, X_{s-}\right)-f\left(Y_{s-} \wedge n, X_{s-}\right)\right)^{2} C_{\alpha} z^{-1-\alpha} \mathrm{d} s \mathrm{~d} z\right) \\
& \quad \leq C_{5} \int_{0}^{t} \mathbb{E}\left(\left(Y_{s} \wedge n\right)^{2 / \alpha}\right) \mathrm{d} s \int_{0}^{1} z^{1-\alpha} \mathrm{d} z \\
& \quad \leq C_{5} n^{2 / \alpha} \frac{t}{2-\alpha} \\
& \quad<\infty, \quad t \in \mathbb{R}_{+},
\end{aligned}
$$

with some constant $C_{5}>0$, by [19, pp. 62, 63], $\left(M_{t}^{3, n}(f)\right)_{t \geq 0}$ is a square-integrable martingale with respect to the filtration $\left(\mathcal{F}_{t}\right)_{t \geq 0}$. This yields the martingale property of $\left(M_{t}^{1}(f)\right)_{t \geq 0}$ with respect to the filtration $\left(\mathcal{F}_{t}\right)_{t \geq 0}$. Indeed, for all $n \in \mathbb{N}$ and $f \in \mathcal{C}_{c}^{2}\left(\mathbb{R}_{+} \times \mathbb{R}, \mathbb{R}\right)$, let

$$
\eta_{t}^{n}(f):=M_{t}^{1}(f)-M_{t}^{2, n}(f)-M_{t}^{3, n}(f), \quad t \in \mathbb{R}_{+} .
$$

Then, by Taylor's theorem,

$$
\begin{aligned}
\eta_{t}^{n}(f) & =\int_{0}^{t} \int_{0}^{\infty} \mathbf{1}_{\left\{Y_{s-}>n\right\}}\left(f\left(Y_{s-}+z \sqrt[\alpha]{Y_{s-}}, X_{s-}\right)-f\left(Y_{s-}, X_{s-}\right)\right) \tilde{N}(\mathrm{~d} s, \mathrm{~d} z) \\
& =\int_{0}^{t} \int_{0}^{\infty} \mathbf{1}_{\left\{Y_{s-}>n\right\}} f_{1}^{\prime}\left(Y_{s-}+\zeta z \sqrt[\alpha]{Y_{s-}}, X_{s-}\right) z \sqrt[\alpha]{Y_{s-}} \tilde{N}(\mathrm{~d} s, \mathrm{~d} z) \\
& =\int_{0}^{t} f_{1}^{\prime}\left(Y_{s-}+\zeta z \sqrt[\alpha]{Y_{s-}}, X_{s-}\right) \sqrt[\alpha]{Y_{s-}} \mathrm{d} L_{s}, \quad t \in \mathbb{R}_{+},
\end{aligned}
$$

with some (not necessarily measurable) function $\zeta: \Omega \rightarrow \mathbb{R}$. Note that, despite the fact that $\zeta$ is not necessarily measurable, $f_{1}^{\prime}\left(Y_{s-}+\zeta z \sqrt[\alpha]{Y_{s-}}, X_{s-}\right)$ is a random variable, since it equals $f\left(Y_{s-}+z \sqrt[\alpha]{Y_{s-}}, X_{s_{-}}\right)-f\left(Y_{s_{-}}, X_{s_{-}}\right)$, which is a random variable. In the same way, 
$\left(f_{1}^{\prime}\left(Y_{s-}+\zeta z \sqrt[\alpha]{Y_{s-}}, X_{s-}\right)\right)_{s \in[0, t]}$ is a predictable process; thus, we can use Lemma 2.8 of [26], and, hence, there exist some constants $C_{6}>0$ and $C_{7}>0$ such that, for all $t \in \mathbb{R}_{+}$,

$$
\begin{aligned}
\mathbb{E}\left(\sup _{s \in[0, t]}\left|\eta_{s}^{n}(f)\right|\right) & \leq C_{6} \mathbb{E}\left(\left(\int_{0}^{t} \mathbf{1}_{\left\{Y_{s}>n\right\}}\left(f_{1}^{\prime}\left(Y_{s}+\zeta z \sqrt[\alpha]{Y_{s}}, X_{S}\right)\right)^{\alpha} Y_{s} \mathrm{~d} s\right)^{1 / \alpha}\right) \\
& \leq C_{7}\left(\int_{0}^{t} \mathbb{E}\left(\mathbf{1}_{\left\{Y_{s}>n\right\}} Y_{s}\right) \mathrm{d} s\right)^{1 / \alpha},
\end{aligned}
$$

where the last inequality follows by Jensen's inequality and the fact that $f^{\prime}$ is bounded. As there exists some constant $C_{8}>0$ such that $\mathbb{E}\left(Y_{t}\right) \leq C_{8}\left(1+y_{0} \mathrm{e}^{-b t / \alpha}\right), t \in \mathbb{R}_{+}$(see, e.g. Formula (2.7) or Proposition 2.10 of [26]), we have

$$
\int_{0}^{t} \mathbb{E}\left(\mathbf{1}_{\left\{Y_{s}>n\right\}} Y_{S}\right) \mathrm{d} s \leq C_{8} \int_{0}^{t}\left(1+y_{0} \mathrm{e}^{-b s / \alpha}\right) \mathrm{d} s<\infty, \quad n \in \mathbb{N},
$$

and so, by the dominated convergence theorem,

$$
\lim _{n \rightarrow \infty} \mathbb{E}\left(\sup _{s \in[0, t]}\left|\eta_{s}^{n}(f)\right|\right)=0, \quad t \in \mathbb{R}_{+} .
$$

Then $\left(M_{t}^{1}(f)\right)_{t \geq 0}$ is a martingale with respect to the filtration $\left(\mathcal{F}_{t}\right)_{t \geq 0}$. For more details, see [3, Proof of Theorem 2.2].

Finally, we check that the transition semigroup $\left(P_{t}\right)_{t \geq 0}$ with state space $\mathbb{R}_{+} \times \mathbb{R}$ corresponding to $\left(Y_{t}, X_{t}\right)_{t \geq 0}$ is a regular affine semigroup having infinitesimal generator given by (2.3) or (2.4) in the $\alpha \in(1,2)$ or, respectively, $\alpha=2$ case. Using the notation given in [12], $\left(0,\left(\alpha_{i, j}\right)_{i, j=1}^{2},\left(b_{i}\right)_{i=1}^{2},\left(\beta_{i, j}\right)_{i, j=1}^{2}, 0, \mu\right)$ and $\left(0,\left(\alpha_{i, j}\right)_{i, j=1}^{2},\left(b_{i}\right)_{i=1}^{2},\left(\beta_{i, j}\right)_{i, j=1}^{2}, 0,0\right)$ are sets of admissible parameters corresponding to the $\alpha \in(1,2)$ and $\alpha=2$ cases, respectively, where

$$
\begin{aligned}
& \left(\begin{array}{ll}
\alpha_{1,1} & \alpha_{1,2} \\
\alpha_{2,1} & \alpha_{2,2}
\end{array}\right):= \begin{cases}\left(\begin{array}{cc}
0 & 0 \\
0 & \frac{1}{2}
\end{array}\right) & \text { if } \alpha \in(1,2), \\
\left(\begin{array}{cc}
\frac{1}{2} & 0 \\
0 & \frac{1}{2}
\end{array}\right) & \text { if } \alpha=2,\end{cases} \\
& \left(b_{1}, b_{2}\right):=(a, m) \text { for } \alpha \in(1,2], \\
& \left(\begin{array}{ll}
\beta_{1,1} & \beta_{1,2} \\
\beta_{2,1} & \beta_{2,2}
\end{array}\right):=\left(\begin{array}{cc}
b & 0 \\
0 & -\theta
\end{array}\right) \quad \text { for } \alpha \in(1,2] \text {, } \\
& \mu(\mathrm{d} y, \mathrm{~d} x):=C_{\alpha} y^{-1-\alpha} \mathrm{d} y \delta_{0}(\mathrm{~d} x) \text { for } \alpha \in(1,2),
\end{aligned}
$$

and $\delta_{0}$ denotes the Dirac measure concentrated on $0 \in \mathbb{R}$. Hence, it follows from Theorem 2.7 of [13] (see also Theorem 6.1 of [12]) that, for these sets of admissible parameters, there exists a regular affine semigroup $\left(Q_{t}\right)_{t \geq 0}$ with infinitesimal generator given by (2.3) or (2.4) in the $\alpha \in(1,2)$ or, respectively, $\alpha=2$ case. By Theorem 2.7 of $[13], \mathcal{C}_{c}^{\infty}\left(\mathbb{R}_{+} \times \mathbb{R}, \mathbb{R}\right)$ is a core of the infinitesimal generator corresponding to the affine semigroup $\left(Q_{t}\right)_{t \geq 0}$. Since we have checked that the infinitesimal generators corresponding to the transition semigroups $\left(P_{t}\right)_{t \geq 0}$ and $\left(Q_{t}\right)_{t \geq 0}$ (defined on the Banach space of bounded functions on $\mathbb{R}_{+} \times \mathbb{R}$ ) coincide on $\mathcal{C}_{c}^{\infty}\left(\mathbb{R}_{+} \times \mathbb{R}, \mathbb{R}\right)$, by the definition of a core, they coincide on the Banach space of bounded functions on $\mathbb{R}_{+} \times \mathbb{R}$. Then $\left(Y_{t}, X_{t}\right)_{t \geq 0}$ is an affine process with infinitesimal generator (2.3) 
or (2.4) in the $\alpha \in(1,2)$ or, respectively, $\alpha=2$ case. We also note that we could have used Lemma 10.2 of [13] to conclude that $\left(Y_{t}, X_{t}\right)_{t \geq 0}$ is a regular affine process with infinitesimal generator (2.3) or (2.4) in the $\alpha \in(1,2)$ or, respectively, $\alpha=2$ case, since we have checked that $\left(M_{t}(f)\right)_{t \geq 0}$ is a martingale with respect to the filtration $\left(\mathscr{F}_{t}\right)_{t \geq 0}$ for any $f \in \mathcal{C}_{c}^{2}\left(\mathbb{R}_{+} \times \mathbb{R}, \mathbb{R}\right)$ in both the $\alpha \in(1,2)$ and $\alpha=2$ cases.

Remark 2.1. In Theorem 2.1 it was assumed that $a>0$, which ensures that $\mathbb{P}\left(Y_{t} \geq 0\right.$ for all $t \geq 0)=1$.

\section{Stationarity}

The study of existence of stationary distributions for affine processes in general is currently under active research.

In the special case of continuous-state branching processes with immigration the question of existence of a unique stationary distribution has been studied; see [23, Theorem 2.6] or [25, Theorem 3.20 and Corollary 3.21].

Glasserman and Kim [17, Theorem 2.4] proved existence of a unique stationary distribution for the process

$$
\begin{gathered}
\mathrm{d} Y_{t}=\left(a-b Y_{t}\right) \mathrm{d} t+\sqrt{Y_{t}} \mathrm{~d} L_{t}, \quad t \geq 0, \\
\mathrm{~d} X_{t}=-\theta X_{t} \mathrm{~d} t+\sqrt{1+\sigma Y_{t}} \mathrm{~d} B_{t}, \quad t \geq 0,
\end{gathered}
$$

where $a>0, b>0, \theta>0, \sigma \geq 0$, and $L$ and $B$ are independent standard Wiener processes.

The following result gives the existence of a unique stationary distribution of the affine process given by SDE (1.1) for both the $\alpha \in(1,2)$ and $\alpha=2$ cases.

Theorem 3.1. Consider the two-dimensional affine model (1.1) with $a>0, b>0, m \in \mathbb{R}$, and $\theta>0$, and with a random initial value $\left(\eta_{0}, \zeta_{0}\right)$ independent of $\left(L_{t}, B_{t}\right)_{t \geq 0}$ satisfying $\mathbb{P}\left(\eta_{0} \geq 0\right)=1$. Then

(i) $\left(Y_{t}, X_{t}\right) \stackrel{\mathrm{D}}{\rightarrow}\left(Y_{\infty}, X_{\infty}\right)$ as $t \rightarrow \infty$, and the distribution of $\left(Y_{\infty}, X_{\infty}\right)$ is given by

$$
\mathbb{E}\left(\mathrm{e}^{-\lambda_{1} Y_{\infty}+\mathrm{i} \lambda_{2} X_{\infty}}\right)=\exp \left\{-a \int_{0}^{\infty} v_{s}\left(\lambda_{1}, \lambda_{2}\right) \mathrm{d} s+\mathrm{i} \frac{m}{\theta} \lambda_{2}\right\}
$$

for $\left(\lambda_{1}, \lambda_{2}\right) \in \mathbb{R}_{+} \times \mathbb{R}$, where $v_{t}\left(\lambda_{1}, \lambda_{2}\right), t \geq 0$, is the unique nonnegative solution of the (deterministic) differential equation

$$
\begin{gathered}
\frac{\partial v_{t}}{\partial t}\left(\lambda_{1}, \lambda_{2}\right)=-b v_{t}\left(\lambda_{1}, \lambda_{2}\right)-\frac{1}{\alpha}\left(v_{t}\left(\lambda_{1}, \lambda_{2}\right)\right)^{\alpha}+\frac{1}{2} \mathrm{e}^{-2 \theta t} \lambda_{2}^{2}, \quad t \geq 0, \\
v_{0}\left(\lambda_{1}, \lambda_{2}\right)=\lambda_{1},
\end{gathered}
$$

(ii) supposing that the random initial value $\left(\eta_{0}, \zeta_{0}\right)$ has the same distribution as $\left(Y_{\infty}, X_{\infty}\right)$ given in part $(i),\left(Y_{t}, X_{t}\right)_{t \geq 0}$ is strictly stationary.

Proof. (i) We prove this part in three steps.

Step 1. In this step we give some representations of the affine transition semigroup $\left(P_{t}\right)_{t \geq 0}$ with state space $\mathbb{R}_{+} \times \mathbb{R}$ corresponding to the process given by SDE (1.1). By Theorem 6.1 of [12] and Theorem 2.1, the transition semigroup $\left(P_{t}\right)_{t \geq 0}$ is given by

$$
\int_{\mathbb{R}_{+} \times \mathbb{R}} \mathrm{e}^{\langle u, \xi\rangle} P_{t}\left(\left(y_{0}, x_{0}\right), \mathrm{d} \xi\right)=\mathrm{e}^{\left\langle\left(y_{0}, x_{0}\right), \psi_{t}(u)\right\rangle+\phi_{t}(u)}
$$


for $u \in U,\left(y_{0}, x_{0}\right) \in \mathbb{R}_{+} \times \mathbb{R}, t \geq 0$, where $U:=\mathrm{C}_{-} \times(\mathrm{i} \mathbb{R})$ with

$$
\mathrm{C}_{-}:=\left\{z_{1}+\mathrm{i} z_{2}: z_{1} \in(-\infty, 0], z_{2} \in \mathbb{R}\right\}, \quad \mathrm{i} \mathbb{R}:=\left\{\mathrm{i} z_{2}: z_{2} \in \mathbb{R}\right\},
$$

and for all $u=\left(u_{1}, u_{2}\right) \in U$, we have $\psi_{t}(u)=\left(\psi_{t}^{(1)}(u), \mathrm{e}^{-\theta t} u_{2}\right), t \geq 0$, where $\psi_{t}^{(1)}(u)$, $t \geq 0$, is a solution of the generalized Riccati equation

$$
\frac{\partial \psi_{t}^{(1)}}{\partial t}(u)=R\left(\psi_{t}^{(1)}(u), \mathrm{e}^{-\theta t} u_{2}\right), \quad t \geq 0, \quad \psi_{0}^{(1)}(u)=u_{1},
$$

and

$$
\phi_{t}(u)=\int_{0}^{t} F\left(\psi_{s}^{(1)}(u), \mathrm{e}^{-\theta s} u_{2}\right) \mathrm{d} s, \quad t \geq 0 .
$$

Here, for $\alpha \in(1,2]$, the (complex-valued) functions $F$ and $R$ are given by

$$
F(u)=a u_{1}+m u_{2}, \quad R(u)=-b u_{1}+\frac{\left(-u_{1}\right)^{\alpha}}{\alpha}+\frac{u_{2}^{2}}{2}, \quad u=\left(u_{1}, u_{2}\right) \in U .
$$

Indeed, in the $\alpha \in(1,2)$ case, the formula for $R(u), u \in U$, can be checked as follows. By [12], in the $\alpha \in(1,2)$ case,

$$
\begin{aligned}
R(u) & :=-b u_{1}+\frac{u_{2}^{2}}{2}+\int_{0}^{\infty} \int_{-\infty}^{\infty}\left(\mathrm{e}^{\langle u, \xi\rangle}-1-\langle u, \xi\rangle\right) C_{\alpha} \xi_{1}^{-1-\alpha} \mathrm{d} \xi_{1} \delta_{0}\left(\mathrm{~d} \xi_{2}\right) \\
& =-b u_{1}+\frac{u_{2}^{2}}{2}+C_{\alpha} \int_{0}^{\infty}\left(\mathrm{e}^{u_{1} \xi_{1}}-1-u_{1} \xi_{1}\right) \xi_{1}^{-1-\alpha} \mathrm{d} \xi_{1} \\
& =-b u_{1}+\frac{\left(-u_{1}\right)^{\alpha}}{\alpha}+\frac{u_{2}^{2}}{2}, \quad u \in U
\end{aligned}
$$

where, for the last equality, we used the facts that $1 / \Gamma(-\alpha)=\alpha(\alpha-1) / \Gamma(2-\alpha)$, the imaginary part of $-\mathrm{i} u_{1} \xi_{1}$ is nonnegative, and

$$
(-\mathrm{i} z)^{\alpha}=\frac{\alpha(\alpha-1)}{\Gamma(2-\alpha)} \int_{0}^{\infty}\left(\mathrm{e}^{\mathrm{i} z \xi_{1}}-1-\mathrm{i} z \xi_{1}\right) \xi_{1}^{-1-\alpha} \mathrm{d} \xi_{1}
$$

for all complex numbers $z$ with nonnegative imaginary part; see, e.g. [35, pp. 67, 68].

Note also that, for all $u=\left(u_{1}, u_{2}\right) \in U$ and $t \geq 0$, the real part of $\psi_{t}^{(1)}(u)$ is less than or equal to 0 (compare also with Remark 2.2 of [13]), and, in addition, if $u_{1} \in \mathbb{R}$ such that $u_{1} \leq 0$ then $\psi_{t}^{(1)}(u) \in \mathbb{R}$ with $\psi_{t}^{(1)}(u) \leq 0$. Moreover, for all $t \geq 0$, we have

$$
\phi_{t}(u)=\int_{0}^{t}\left(a \psi_{s}^{(1)}(u)+m \mathrm{e}^{-\theta s} u_{2}\right) \mathrm{d} s=a \int_{0}^{t} \psi_{s}^{(1)}(u) \mathrm{d} s+m u_{2} \frac{1-\mathrm{e}^{-\theta t}}{\theta} .
$$

In fact, one can give a simplified characterization of the transition semigroup $\left(P_{t}\right)_{t \geq 0}$ by

$$
\begin{aligned}
& \int_{0}^{\infty} \int_{-\infty}^{\infty} \mathrm{e}^{-\lambda_{1} \xi_{1}+\mathrm{i} \lambda_{2} \xi_{2}} P_{t}\left(\left(y_{0}, x_{0}\right), \mathrm{d} \xi_{1}, \mathrm{~d} \xi_{2}\right) \\
& \quad=\exp \left\{-y_{0} v_{t}\left(\lambda_{1}, \lambda_{2}\right)+\mathrm{i} x_{0} \mathrm{e}^{-\theta t} \lambda_{2}+g_{t}\left(\lambda_{1}, \lambda_{2}\right)\right\}
\end{aligned}
$$


for $\left(\lambda_{1}, \lambda_{2}\right),\left(y_{0}, x_{0}\right) \in \mathbb{R}_{+} \times \mathbb{R}$, where

$$
\begin{aligned}
g_{t}\left(\lambda_{1}, \lambda_{2}\right) & :=\int_{0}^{t}\left(-a v_{s}\left(\lambda_{1}, \lambda_{2}\right)+\mathrm{i} m \mathrm{e}^{-\theta s} \lambda_{2}\right) \mathrm{d} s \\
& =-a \int_{0}^{t} v_{s}\left(\lambda_{1}, \lambda_{2}\right) \mathrm{d} s+\mathrm{i} m \lambda_{2} \frac{1-\mathrm{e}^{-\theta t}}{\theta}
\end{aligned}
$$

and $v_{t}\left(\lambda_{1}, \lambda_{2}\right), t \geq 0$, is the unique, nonnegative solution of the differential equation

$$
\begin{gathered}
\frac{\partial v_{t}}{\partial t}\left(\lambda_{1}, \lambda_{2}\right)=-b v_{t}\left(\lambda_{1}, \lambda_{2}\right)-\frac{1}{\alpha}\left(v_{t}\left(\lambda_{1}, \lambda_{2}\right)\right)^{\alpha}+\frac{1}{2} \mathrm{e}^{-2 \theta t} \lambda_{2}^{2}, \quad t \geq 0, \\
v_{0}\left(\lambda_{1}, \lambda_{2}\right)=\lambda_{1},
\end{gathered}
$$

in the $\alpha \in(1,2]$ case; see [3, Proof of Theorem 3.1]. Note also that $v_{t}\left(\lambda_{1}, \lambda_{2}\right) \geq 0$ for all $\left(\lambda_{1}, \lambda_{2}\right) \in \mathbb{R}_{+} \times \mathbb{R}$, since $\psi_{t}^{(1)}(u) \leq 0$ for $u_{1} \leq 0$. The uniqueness of the solutions of the differential equation (Cauchy problem) (3.5) follows by general results of [13, Propositions 6.1 and 6.4, and Lemma 9.2]. For a direct proof, see the proof of Theorem 3.1 of [3].

Step 2. We show that

$$
v_{t}\left(\lambda_{1}, \lambda_{2}\right) \leq M\left(\lambda_{1}, \lambda_{2}\right)(1+t) \max \left(\mathrm{e}^{-2 \theta t}, \mathrm{e}^{-b t}\right), \quad t \geq 0,\left(\lambda_{1}, \lambda_{2}\right) \in \mathbb{R}_{+} \times \mathbb{R},
$$

where

$$
M\left(\lambda_{1}, \lambda_{2}\right):= \begin{cases}\lambda_{1}+\frac{\lambda_{2}^{2}}{2|b-2 \theta|} & \text { if } b \neq 2 \theta \\ \lambda_{1}+\frac{\lambda_{2}^{2}}{2} & \text { if } b=2 \theta .\end{cases}
$$

The proof is based on the following version of the comparison theorem (see, e.g. [34] or Lemma B.3. of [15]): if $S: \mathbb{R}_{+} \times \mathbb{R} \rightarrow \mathbb{R}$ is a continuous function which is locally Lipschitz continuous in its second variable and $p, q: \mathbb{R}_{+} \rightarrow \mathbb{R}$ are differentiable functions satisfying

$$
p^{\prime}(t) \leq S(t, p(t)), \quad t \geq 0, \quad q^{\prime}(t)=S(t, q(t)), \quad t \geq 0, \quad p(0) \leq q(0),
$$

then $p(t) \leq q(t)$ for all $t \geq 0$. Using this, we can check that $v_{t}\left(\lambda_{1}, \lambda_{2}\right) \leq u_{t}\left(\lambda_{1}, \lambda_{2}\right)$ for all $t \geq 0$ and $\left(\lambda_{1}, \lambda_{2}\right) \in \mathbb{R}_{+} \times \mathbb{R}$, where, for all $\left(\lambda_{1}, \lambda_{2}\right) \in \mathbb{R}_{+} \times \mathbb{R}, u_{t}\left(\lambda_{1}, \lambda_{2}\right), t \geq 0$, is the unique solution of the differential equation

$$
\frac{\partial u_{t}}{\partial t}\left(\lambda_{1}, \lambda_{2}\right)=-b u_{t}\left(\lambda_{1}, \lambda_{2}\right)+\frac{1}{2} \mathrm{e}^{-2 \theta t} \lambda_{2}^{2}, \quad t \geq 0, \quad u_{0}\left(\lambda_{1}, \lambda_{2}\right)=\lambda_{1} .
$$

Furthermore, we can verify that

$$
u_{t}\left(\lambda_{1}, \lambda_{2}\right)= \begin{cases}\left(\lambda_{1}+\frac{\lambda_{2}^{2}}{2(-b+2 \theta)}\right) \mathrm{e}^{-b t}-\frac{\lambda_{2}^{2}}{2(-b+2 \theta)} \mathrm{e}^{-2 \theta t} & \text { if } b \neq 2 \theta \\ \left(\lambda_{1}+\frac{\lambda_{2}^{2}}{2} t\right) \mathrm{e}^{-b t} & \text { if } b=2 \theta\end{cases}
$$

For more details, see the proof of Theorem 3.1 of [3].

Finally, by (3.7), (3.6) readily holds for $b=2 \theta$. If $b>2 \theta$ then

$$
u_{t}\left(\lambda_{1}, \lambda_{2}\right) \leq \lambda_{1} \mathrm{e}^{-2 \theta t}-\frac{\lambda_{2}^{2}}{2(-b+2 \theta)} \mathrm{e}^{-2 \theta t} \leq\left(\lambda_{1}-\frac{\lambda_{2}^{2}}{2(-b+2 \theta)}\right)(1+t) \mathrm{e}^{-2 \theta t},
$$


and if $0<b<2 \theta$ then

$$
u_{t}\left(\lambda_{1}, \lambda_{2}\right) \leq\left(\lambda_{1}+\frac{\lambda_{2}^{2}}{2(-b+2 \theta)}\right) \mathrm{e}^{-b t} \leq\left(\lambda_{1}+\frac{\lambda_{2}^{2}}{2(-b+2 \theta)}\right)(1+t) \mathrm{e}^{-b t},
$$

as desired.

Step 3. By the continuity theorem and (3.3), to prove (i), it is enough to check that, for all $\left(\lambda_{1}, \lambda_{2}\right),\left(y_{0}, x_{0}\right) \in \mathbb{R}_{+} \times \mathbb{R}$,

$$
\begin{aligned}
\lim _{t \rightarrow \infty}\left(-y_{0} v_{t}\left(\lambda_{1}, \lambda_{2}\right)+\mathrm{i} x_{0} \mathrm{e}^{-\theta t} \lambda_{2}+g_{t}\left(\lambda_{1}, \lambda_{2}\right)\right) & =-a \int_{0}^{\infty} v_{s}\left(\lambda_{1}, \lambda_{2}\right) \mathrm{d} s+\mathrm{i} \frac{m}{\theta} \lambda_{2} \\
& =: g_{\infty}\left(\lambda_{1}, \lambda_{2}\right),
\end{aligned}
$$

and that the function $\mathbb{R}_{+} \times \mathbb{R} \ni\left(\lambda_{1}, \lambda_{2}\right) \mapsto g_{\infty}\left(\lambda_{1}, \lambda_{2}\right)$ is continuous. Indeed, using (3.3), and the independence of $\left(\eta_{0}, \zeta_{0}\right)$ and $\left(L_{t}, B_{t}\right)_{t \geq 0}$, the law of total expectation yields

$$
\begin{aligned}
\mathbb{E}\left(\mathrm{e}^{-\lambda_{1} Y_{t}+\mathrm{i} \lambda_{2} X_{t}}\right) & \\
& =\int_{0}^{\infty} \int_{-\infty}^{\infty} \mathbb{E}\left(\mathrm{e}^{-\lambda_{1} Y_{t}+\mathrm{i} \lambda_{2} X_{t}} \mid\left(Y_{0}, X_{0}\right)=\left(y_{0}, x_{0}\right)\right) \mathbb{P}_{\left(Y_{0}, X_{0}\right)}\left(\mathrm{d} y_{0}, \mathrm{~d} x_{0}\right) \\
& =\int_{0}^{\infty} \int_{-\infty}^{\infty} \exp \left\{-y_{0} v_{t}\left(\lambda_{1}, \lambda_{2}\right)+\mathrm{i} x_{0} \mathrm{e}^{-\theta t} \lambda_{2}+g_{t}\left(\lambda_{1}, \lambda_{2}\right)\right\} \mathbb{P}_{\left(Y_{0}, X_{0}\right)}\left(\mathrm{d} y_{0}, \mathrm{~d} x_{0}\right)
\end{aligned}
$$

for all $\left(\lambda_{1}, \lambda_{2}\right) \in \mathbb{R}_{+} \times \mathbb{R}$, where $\mathbb{P}_{\left(Y_{0}, X_{0}\right)}$ denotes the distribution of $\left(Y_{0}, X_{0}\right)$ on $\mathbb{R}_{+} \times \mathbb{R}$, and, hence, (3.8) and the dominated convergence theorem imply that

$$
\lim _{t \rightarrow \infty} \mathbb{E}\left(\mathrm{e}^{-\lambda_{1} Y_{t}+\mathrm{i} \lambda_{2} X_{t}}\right)=\int_{0}^{\infty} \int_{-\infty}^{\infty} \mathrm{e}^{g_{\infty}\left(\lambda_{1}, \lambda_{2}\right)} \mathbb{P}_{\left(Y_{0}, X_{0}\right)}\left(\mathrm{d} y_{0}, \mathrm{~d} x_{0}\right)=\mathrm{e}^{g_{\infty}\left(\lambda_{1}, \lambda_{2}\right)}
$$

for $\left(\lambda_{1}, \lambda_{2}\right) \in \mathbb{R}_{+} \times \mathbb{R}$. Then, using the continuity of the function $\mathbb{R}_{+} \times \mathbb{R} \ni\left(\lambda_{1}, \lambda_{2}\right) \mapsto$ $g_{\infty}\left(\lambda_{1}, \lambda_{2}\right)$ (which will be checked later), the continuity theorem yields (i).

Now we prove (3.8). Since $\theta>0$ and $b>0$, by (3.6), and using the fact that $v_{t}\left(\lambda_{1}, \lambda_{2}\right) \geq 0$ for all $t \geq 0$ and $\left(\lambda_{1}, \lambda_{2}\right) \in \mathbb{R}_{+} \times \mathbb{R}$ (see step 1$)$, we have

$$
\lim _{t \rightarrow \infty}\left(-y_{0} v_{t}\left(\lambda_{1}, \lambda_{2}\right)+\mathrm{i} x_{0} \mathrm{e}^{-\theta t} \lambda_{2}\right)=0
$$

Recall that

$$
g_{t}\left(\lambda_{1}, \lambda_{2}\right)=-a \int_{0}^{t} v_{s}\left(\lambda_{1}, \lambda_{2}\right) \mathrm{d} s+\mathrm{i} m \lambda_{2} \frac{1-\mathrm{e}^{-\theta t}}{\theta} .
$$

Since $\theta>0$, we have $\lim _{t \rightarrow \infty}\left(1-\mathrm{e}^{-\theta t}\right) / \theta=1 / \theta$, and since $v_{t}\left(\lambda_{1}, \lambda_{2}\right) \geq 0$ for all $t \geq 0$, $\left(\lambda_{1}, \lambda_{2}\right) \in \mathbb{R}_{+} \times \mathbb{R}$ (see step 1), by the dominated convergence theorem and (3.6), we obtain

$$
\lim _{t \rightarrow \infty} \int_{0}^{t} v_{s}\left(\lambda_{1}, \lambda_{2}\right) \mathrm{d} s=\int_{0}^{\infty} v_{s}\left(\lambda_{1}, \lambda_{2}\right) \mathrm{d} s .
$$

Indeed, for all $t \geq 0$ and $s \geq 0,\left|v_{s}\left(\lambda_{1}, \lambda_{2}\right) \mathbf{1}_{[0, t]}(s)\right| \leq v_{s}\left(\lambda_{1}, \lambda_{2}\right)$, and, by (3.6),

$$
\begin{aligned}
\int_{0}^{\infty} v_{s}\left(\lambda_{1}, \lambda_{2}\right) \mathrm{d} s & \leq M\left(\lambda_{1}, \lambda_{2}\right) \int_{0}^{\infty}(1+s) \max \left(\mathrm{e}^{-2 \theta s}, \mathrm{e}^{-b s}\right) \mathrm{d} s \\
& =M\left(\lambda_{1}, \lambda_{2}\right) \max \left(\frac{1}{b}+\frac{1}{b^{2}}, \frac{1}{2 \theta}+\frac{1}{(2 \theta)^{2}}\right) \\
& <\infty .
\end{aligned}
$$


The continuity of the function $\mathbb{R}_{+} \times \mathbb{R} \ni\left(\lambda_{1}, \lambda_{2}\right) \mapsto g_{\infty}\left(\lambda_{1}, \lambda_{2}\right)$ can be checked as follows. It will follow if we prove that, for all $s \geq 0$, the function $v_{s}$ is continuous. Namely, if $\lambda^{(n)}=\left(\lambda_{1}^{(n)}, \lambda_{2}^{(n)}\right), n \in \mathbb{N}$, is a sequence in $\mathbb{R}_{+} \times \mathbb{R}$ such that $\lim _{n \rightarrow \infty} \lambda^{(n)}=\lambda$, where $\lambda \in \mathbb{R}_{+} \times \mathbb{R}$, then $\lim _{n \rightarrow \infty} v_{s}\left(\lambda^{(n)}\right)=v_{s}(\lambda)$ for all $s \geq 0$, and, by (3.6),

$$
v_{s}\left(\lambda^{(n)}\right)=v_{s}\left(\lambda_{1}^{(n)}, \lambda_{2}^{(n)}\right) \leq M\left(\lambda_{1}^{(n)}, \lambda_{2}^{(n)}\right)(1+s) \max \left(\mathrm{e}^{-2 \theta s}, \mathrm{e}^{-b s}\right), \quad n \in \mathbb{N}, s \geq 0 .
$$

Since the sequence $\lambda^{(n)}, n \in \mathbb{N}$, is bounded (since it is convergent), we have $\sup _{n \in \mathbb{N}} M\left(\lambda_{1}^{(n)}\right.$, $\left.\lambda_{2}^{(n)}\right)<\infty$, and using also that $\int_{0}^{\infty}(1+s) \max \left(\mathrm{e}^{-2 \theta s}, \mathrm{e}^{-b s}\right) \mathrm{d} s<\infty$, the dominated convergence theorem implies that

$$
\lim _{n \rightarrow \infty} \int_{0}^{\infty} v_{s}\left(\lambda_{1}^{(n)}, \lambda_{2}^{(n)}\right) \mathrm{d} s=\int_{0}^{\infty} v_{s}\left(\lambda_{1}, \lambda_{2}\right) \mathrm{d} s
$$

which shows the continuity of $g_{\infty}$. Finally, we prove that, for all $s \geq 0$, the function $\mathbb{R}_{+} \times \mathbb{R} \ni$ $\left(\lambda_{1}, \lambda_{2}\right) \mapsto v_{s}\left(\lambda_{1}, \lambda_{2}\right)$ is continuous. Note that the function $v_{s}$ does not depend on the parameters $a$ and $m$, since it is the unique solution of the differential equation (3.2). Let $\left(\widetilde{Y}_{t}, \widetilde{X}_{t}\right)_{t \geq 0}$ be an affine process satisfying $\operatorname{SDE}(1.1)$ with initial value $\left(\widetilde{Y}_{0}, \widetilde{X}_{0}\right)=\left(Y_{0}, X_{0}\right)$, and with parameters $a=m=0$ and the given $b>0$ and $\theta>0$. Then, by (3.3),

$$
\int_{0}^{\infty} \int_{-\infty}^{\infty} \mathrm{e}^{-\lambda_{1} \xi_{1}+\mathrm{i} \lambda_{2} \xi_{2}} \widetilde{P}_{s}\left(\left(y_{0}, x_{0}\right), \mathrm{d} \xi_{1}, \mathrm{~d} \xi_{2}\right)=\exp \left\{-y_{0} v_{s}\left(\lambda_{1}, \lambda_{2}\right)+\mathrm{i} x_{0} \mathrm{e}^{-\theta s} \lambda_{2}\right\}
$$

for $s \in \mathbb{R}_{+}$and $\left.\left(\lambda_{1}, \lambda_{2}\right), \underset{\widetilde{Y}}{\left(y_{0}\right.}, x_{0}\right) \in \mathbb{R}_{+} \times \mathbb{R}$, where $\left(\widetilde{P}_{t}\right)_{t \geq 0}$ denotes the transition semigroup of the affine process $\left(\widetilde{Y}_{t}, \widetilde{X}_{t}\right)_{t \geq 0}$. For all $s \in \mathbb{R}_{+}$, the left-hand side of (3.9) is a continuous function of $\left(\lambda_{1}, \lambda_{2}\right) \in \mathbb{R}_{+} \times \mathbb{R}$, since,for all $\left(\lambda_{1}, \lambda_{2}\right) \in \mathbb{R}_{+} \times \mathbb{R}$ and $\left(h_{1}, h_{2}\right) \in \mathbb{R}^{2}$,

$$
\begin{aligned}
& \mid \int_{0}^{\infty} \int_{-\infty}^{\infty} \mathrm{e}^{-\left(\lambda_{1}+h_{1}\right) \xi_{1}+\mathrm{i}\left(\lambda_{2}+h_{2}\right) \xi_{2}} \widetilde{P}_{S}\left(\left(y_{0}, x_{0}\right), \mathrm{d} \xi_{1}, \mathrm{~d} \xi_{2}\right) \\
& -\int_{0}^{\infty} \int_{-\infty}^{\infty} \mathrm{e}^{-\lambda_{1} \xi_{1}+\mathrm{i} \lambda_{2} \xi_{2}} \widetilde{P}_{S}\left(\left(y_{0}, x_{0}\right), \mathrm{d} \xi_{1}, \mathrm{~d} \xi_{2}\right) \mid \\
& \quad \leq \int_{0}^{\infty} \int_{-\infty}^{\infty}\left|\mathrm{e}^{-h_{1} \xi_{1}+\mathrm{i} h_{2} \xi_{2}}-1\right| \widetilde{P}_{S}\left(\left(y_{0}, x_{0}\right), \mathrm{d} \xi_{1}, \mathrm{~d} \xi_{2}\right),
\end{aligned}
$$

which tends to 0 as $\left(h_{1}, h_{2}\right) \rightarrow(0,0)$, by the dominated convergence theorem. This implies that the right-hand side of (3.9) is also a continuous function of $\left(\lambda_{1}, \lambda_{2}\right) \in \mathbb{R}_{+} \times \mathbb{R}$. This readily yields the continuity of the function $v_{s}$ for all fixed $s \in \mathbb{R}_{+}$.

(ii) First we check that the one-dimensional distributions of $\left(Y_{t}, X_{t}\right)_{t \geq 0}$ are translation invariant and have the same distribution as $\left(Y_{\infty}, X_{\infty}\right)$. Using (3.1), (3.3), the tower rule, and the independence of $\left(Y_{0}, X_{0}\right)$ and $(L, B)$, it is enough to check that, for all $t \geq 0$ and $\left(\lambda_{1}, \lambda_{2}\right) \in \mathbb{R}_{+} \times \mathbb{R}$

$$
\begin{gathered}
\mathbb{E}\left(\exp \left\{-v_{t}\left(\lambda_{1}, \lambda_{2}\right) Y_{\infty}+\mathrm{ie}^{-\theta t} \lambda_{2} X_{\infty}+g_{t}\left(\lambda_{1}, \lambda_{2}\right)\right\}\right) \\
=\exp \left\{-a \int_{0}^{\infty} v_{s}\left(\lambda_{1}, \lambda_{2}\right) \mathrm{d} s+\mathrm{i} \frac{m}{\theta} \lambda_{2}\right\}
\end{gathered}
$$

By (3.1) and (3.4), and using the fact that $v_{t}\left(\lambda_{1}, \lambda_{2}\right) \geq 0$ for all $t \geq 0$ and $\left(\lambda_{1}, \lambda_{2}\right) \in \mathbb{R}_{+} \times \mathbb{R}$ 
(see step 1 of the proof of part (i)), we have

$$
\begin{aligned}
& \mathbb{E}\left(\exp \left\{-v_{t}\left(\lambda_{1}, \lambda_{2}\right) Y_{\infty}+\mathrm{ie}^{-\theta t} \lambda_{2} X_{\infty}+g_{t}\left(\lambda_{1}, \lambda_{2}\right)\right\}\right) \\
& \quad=\exp \left\{-a \int_{0}^{\infty} v_{s}\left(v_{t}\left(\lambda_{1}, \lambda_{2}\right), \mathrm{e}^{-\theta t} \lambda_{2}\right) \mathrm{d} s+\mathrm{i} \frac{m}{\theta} \mathrm{e}^{-\theta t} \lambda_{2}+g_{t}\left(\lambda_{1}, \lambda_{2}\right)\right\} \\
& \quad=\exp \left\{-a\left(\int_{0}^{\infty} v_{s}\left(v_{t}\left(\lambda_{1}, \lambda_{2}\right), \mathrm{e}^{-\theta t} \lambda_{2}\right) \mathrm{d} s+\int_{0}^{t} v_{s}\left(\lambda_{1}, \lambda_{2}\right) \mathrm{d} s\right)+\mathrm{i} \frac{m}{\theta} \lambda_{2}\right\} .
\end{aligned}
$$

Hence, it remains to check that

$$
\int_{0}^{\infty} v_{s}\left(\lambda_{1}, \lambda_{2}\right) \mathrm{d} s=\int_{0}^{\infty} v_{s}\left(v_{t}\left(\lambda_{1}, \lambda_{2}\right), \mathrm{e}^{-\theta t} \lambda_{2}\right) \mathrm{d} s+\int_{0}^{t} v_{s}\left(\lambda_{1}, \lambda_{2}\right) \mathrm{d} s
$$

for $t \geq 0$ and $\left(\lambda_{1}, \lambda_{2}\right) \in \mathbb{R}_{+} \times \mathbb{R}$, i.e.

$$
\int_{t}^{\infty} v_{s}\left(\lambda_{1}, \lambda_{2}\right) \mathrm{d} s=\int_{0}^{\infty} v_{s}\left(v_{t}\left(\lambda_{1}, \lambda_{2}\right), \mathrm{e}^{-\theta t} \lambda_{2}\right) \mathrm{d} s, \quad t \geq 0,\left(\lambda_{1}, \lambda_{2}\right) \in \mathbb{R}_{+} \times \mathbb{R} .
$$

For this, it is enough to check that

$$
v_{s}\left(v_{t}\left(\lambda_{1}, \lambda_{2}\right), \mathrm{e}^{-\theta t} \lambda_{2}\right)=v_{s+t}\left(\lambda_{1}, \lambda_{2}\right), \quad s, t \geq 0,\left(\lambda_{1}, \lambda_{2}\right) \in \mathbb{R}_{+} \times \mathbb{R},
$$

or, equivalently,

$$
v_{t}\left(v_{s}\left(\lambda_{1}, \lambda_{2}\right), \mathrm{e}^{-\theta s} \lambda_{2}\right)=v_{s+t}\left(\lambda_{1}, \lambda_{2}\right), \quad s, t \geq 0,\left(\lambda_{1}, \lambda_{2}\right) \in \mathbb{R}_{+} \times \mathbb{R} .
$$

By (3.2), we have

$$
\frac{\partial v_{s+t}}{\partial t}\left(\lambda_{1}, \lambda_{2}\right)=-b v_{s+t}\left(\lambda_{1}, \lambda_{2}\right)-\frac{1}{\alpha}\left(v_{s+t}\left(\lambda_{1}, \lambda_{2}\right)\right)^{\alpha}+\frac{1}{2} \mathrm{e}^{-2 \theta(s+t)} \lambda_{2}^{2}, \quad t \geq 0,
$$

with initial condition $v_{s+0}\left(\lambda_{1}, \lambda_{2}\right)=v_{s}\left(\lambda_{1}, \lambda_{2}\right)$. Note also that, again by (3.2),

$$
\begin{aligned}
\frac{\partial v_{t}}{\partial t}\left(v_{s}\left(\lambda_{1}, \lambda_{2}\right), \mathrm{e}^{-\theta s} \lambda_{2}\right)= & -b v_{t}\left(v_{s}\left(\lambda_{1}, \lambda_{2}\right), \mathrm{e}^{-\theta s} \lambda_{2}\right)-\frac{1}{\alpha}\left(v_{t}\left(v_{s}\left(\lambda_{1}, \lambda_{2}\right), \mathrm{e}^{-\theta s} \lambda_{2}\right)\right)^{\alpha} \\
& +\frac{1}{2} \mathrm{e}^{-2 \theta t}\left(\mathrm{e}^{-\theta s} \lambda_{2}\right)^{2}, \quad t \geq 0,
\end{aligned}
$$

with initial condition $v_{0}\left(v_{s}\left(\lambda_{1}, \lambda_{2}\right), \mathrm{e}^{-\theta s} \lambda_{2}\right)=v_{s}\left(\lambda_{1}, \lambda_{2}\right)$. Hence, for all $s \geq 0$, the left- and right-hand sides of (3.10), as functions of $t \geq 0$, satisfy the differential equation (3.2) with the initial value $v_{s}\left(\lambda_{1}, \lambda_{2}\right)$. Since (3.2) has a unique solution for all nonnegative initial values, we obtain (3.10).

Finally, the strict stationarity (translation invariance of the finite-dimensional distributions) of $\left(Y_{t}, X_{t}\right)_{t \geq 0}$ follows by the chain rule for conditional expectations and the fact that it is a time-homogeneous Markov process.

\section{Ergodicity}

As with the existence of a unique stationary distribution, the question of ergodicity for an affine process is also the focus of current investigations. Recently, Sandrić [31] proved ergodicity of so-called stable-like processes using the same technique that we applied. Furthermore, the ergodicity of the so-called $\alpha$-root process with $\alpha \in(1,2]$ (see the first SDE of (1.1)) and some statistical applications were given in [26].

In the following result we give the ergodicity of the affine diffusion process given by SDE (1.1) with $\alpha=2$. 
Theorem 4.1. Consider the two-dimensional affine diffusion model (1.1) with $\alpha=2, a>0$, $b>0, m \in \mathbb{R}$, and $\theta>0$, and with a random initial value $\left(\eta_{0}, \zeta_{0}\right)$ independent of $\left(L_{t}, B_{t}\right)_{t \geq 0}$ satisfying $\mathbb{P}\left(\eta_{0} \geq 0\right)=1$. Then, for all Borel measurable functions $f: \mathbb{R}^{2} \rightarrow \mathbb{R}$ such that $\mathbb{E}\left|f\left(Y_{\infty}, X_{\infty}\right)\right|<\infty$, we have

$$
\mathbb{P}\left(\lim _{T \rightarrow \infty} \frac{1}{T} \int_{0}^{T} f\left(Y_{S}, X_{S}\right) \mathrm{d} s=\mathbb{E} f\left(Y_{\infty}, X_{\infty}\right)\right)=1,
$$

where the distribution of $\left(Y_{\infty}, X_{\infty}\right)$ is given by (3.1) and (3.2) with $\alpha=2$.

Proof. We use the notation of [28] and [29]. Using Theorem 6.1 (so-called Foster-Lyapunov criteria) of [29], it is enough to check that

(a) $\left(Y_{t}, X_{t}\right)_{t \geq 0}$ is a right process (defined as [32, p. 38]);

(b) all compact sets are petite for some skeleton chain (skeleton chains and petite sets are defined in [28, pp. 491, 500] and [27, p. 550], respectively);

(c) there exist $c, d \in \mathbb{R}$ with $c>0$ such that the inequality

$$
\left(\mathcal{A}_{n} V\right)(y, x) \leq-c V(y, x)+d, \quad(y, x) \in O_{n},
$$

holds for all $n \in \mathbb{N}$, where $O_{n}:=\left\{(y, x) \in \mathbb{R}_{+} \times \mathbb{R}:\|(y, x)\|<n\right\}$ for each $n \in \mathbb{N}$,

$$
V(y, x):=\left(y-c_{1}\right)^{2}+\left(x-c_{2}\right)^{2}, \quad(y, x) \in \mathbb{R}_{+} \times \mathbb{R},
$$

with some appropriate $c_{1}, c_{2} \in \mathbb{R}$, and $\mathcal{A}_{n}$ denotes the extended generator of the process $\left(Y_{t}^{(n)}, X_{t}^{(n)}\right)_{t \geq 0}$ given by

$$
\left(Y_{t}^{(n)}, X_{t}^{(n)}\right):= \begin{cases}\left(Y_{t}, X_{t}\right) & \text { for } t<T_{n}, \\ (0, n) & \text { for } t \geq T_{n},\end{cases}
$$

where the stopping time $T_{n}$ is defined by $T_{n}:=\inf \left\{t \in \mathbb{R}_{+}:\left(Y_{t}, X_{t}\right) \in\left(\mathbb{R}_{+} \times \mathbb{R}\right) \backslash O_{n}\right\}$. (Here we note that instead of $(0, n)$ we could have chosen any fixed state in $\left(\mathbb{R}_{+} \times \mathbb{R}\right) \backslash O_{n}$, and we could also have defined $\left(Y_{t}^{(n)}, X_{t}^{(n)}\right)_{t \geq 0}$ as the stopped process $\left(Y_{t \wedge T_{n}}, X_{t \wedge T_{n}}\right)_{t \geq 0}$; see $[29$, p. 521].)

Indeed, Theorem 6.1 of [29] then yields the exponential ergodicity of the process $\left(Y_{t}, X_{t}\right)_{t \in \mathbb{R}_{+}}$, namely, there exist $\beta>0$ and $B \in \mathbb{R}_{+}$such that

$$
\sup _{|g| \leq V+1}\left|\mathbb{E}\left(g\left(Y_{t}, X_{t}\right) \mid\left(Y_{0}, X_{0}\right)=\left(y_{0}, x_{0}\right)\right)-\mathbb{E}\left(g\left(Y_{\infty}, X_{\infty}\right)\right)\right| \leq B\left(V\left(y_{0}, x_{0}\right)+1\right) \mathrm{e}^{-\beta t}
$$

for all $t \in \mathbb{R}_{+}$and $\left(y_{0}, x_{0}\right) \in \mathbb{R}_{+} \times \mathbb{R}$, where the supremum is taken over Borel measurable functions $g: \mathbb{R}_{+} \times \mathbb{R} \rightarrow \mathbb{R}$. According to the discussion after Proposition 2.5 of [6], this implies (4.1). Here we point out that, due to Bhattacharya [6], we do not have to assume that $\mathbb{P}\left(Y_{0}>0\right)=1$ in order to prove (4.1).

To prove (a), it is enough to show that the process $\left(Y_{t}, X_{t}\right)_{t \in \mathbb{R}_{+}}$is a (weak) Feller (see [28, Section 3.1]), strong Markov process with continuous sample paths; see, e.g. [28, p. 498]. According to Proposition 8.2 (or Theorem 2.7) of [13], the process $\left(Y_{t}, X_{t}\right)_{t \geq 0}$ is a Feller Markov process. Since $\left(Y_{t}, X_{t}\right)_{t \geq 0}$ has continuous sample paths almost surely (specifically, it is càdlàg), it is automatically a strong Markov process; see, e.g. Theorem 1 of $[9$, p. 56]. 
To prove (b), in view of Proposition 6.2.8 of [30], it is sufficient to show that the skeleton chain $\left(Y_{n}, X_{n}\right)_{n \in \mathbb{Z}_{+}}$is irreducible with respect to the Lebesgue measure on $\mathbb{R}_{+} \times \mathbb{R}$ (see, e.g. [29, p. 520]), and admits the Feller property. The skeleton chain $\left(Y_{n}, X_{n}\right)_{n \in \mathbb{Z}_{+}}$admits the Feller property since the process $\left(Y_{t}, X_{t}\right)_{t \geq 0}$ is a Feller process. In order to check irreducibility of the skeleton chain $\left(Y_{n}, X_{n}\right)_{n \in \mathbb{Z}_{+}}$with respect to the Lebesgue measure on $\mathbb{R}_{+} \times \mathbb{R}$, it is enough to prove that the conditional distribution of $\left(Y_{1}, X_{1}\right)$ given $\left(Y_{0}, X_{0}\right)$ is absolutely continuous (with respect to the Lebesgue measure on $\mathbb{R}_{+} \times \mathbb{R}$ ) with a conditional density function $f_{\left(Y_{1}, X_{1}\right) \mid\left(Y_{0}, X_{0}\right)}: \mathbb{R}^{2} \times \mathbb{R}^{2} \rightarrow \mathbb{R}_{+}$such that $f_{\left(Y_{1}, X_{1}\right) \mid\left(Y_{0}, X_{0}\right)}\left(y, x \mid y_{0}, x_{0}\right)>0$ for all $\left(y, x, y_{0}, x_{0}\right) \in(0, \infty) \times \mathbb{R} \times \mathbb{R}_{+} \times \mathbb{R}$. Indeed, the Lebesgue measure on $\mathbb{R}_{+} \times \mathbb{R}$ is $\sigma$-finite, and if $B$ is a Borel set in $\mathbb{R}_{+} \times \mathbb{R}$ with positive Lebesgue measure then

$$
\begin{aligned}
\mathbb{E}\left(\sum_{n=0}^{\infty} \mathbf{1}_{B}\left(Y_{n}, X_{n}\right) \mid\left(Y_{0}, X_{0}\right)=\left(y_{0}, x_{0}\right)\right) & \geq \mathbb{P}\left(\left(Y_{1}, X_{1}\right) \in B \mid\left(Y_{0}, X_{0}\right)=\left(y_{0}, x_{0}\right)\right) \\
& =\iint_{B} f_{\left(Y_{1}, X_{1}\right) \mid\left(Y_{0}, X_{0}\right)}\left(y, x \mid y_{0}, x_{0}\right) \mathrm{d} y \mathrm{~d} x \\
& >0
\end{aligned}
$$

for all $\left(y_{0}, x_{0}\right) \in \mathbb{R}_{+} \times \mathbb{R}$. The existence of $f_{\left(Y_{1}, X_{1}\right) \mid\left(Y_{0}, X_{0}\right)}$ with the required property can be checked as follows. By Theorem 2.1 we have

$$
\begin{aligned}
& Y_{1}=\mathrm{e}^{-b}\left(y_{0}+a \int_{0}^{1} \mathrm{e}^{b u} \mathrm{~d} u+\int_{0}^{1} \mathrm{e}^{b u} \sqrt{Y_{u}} \mathrm{~d} L_{u}\right), \\
& X_{1}=\mathrm{e}^{-\theta}\left(x_{0}+m \int_{0}^{1} \mathrm{e}^{\theta u} \mathrm{~d} u+\int_{0}^{1} \mathrm{e}^{\theta u} \sqrt{Y_{u}} \mathrm{~d} B_{u}\right),
\end{aligned}
$$

provided that $\left(Y_{0}, X_{0}\right)=\left(y_{0}, x_{0}\right),\left(y_{0}, x_{0}\right) \in \mathbb{R}_{+} \times \mathbb{R}$. Recall that a two-dimensional random vector $\zeta$ is absolutely continuous if and only if $V \zeta+v$ is absolutely continuous for all invertable matrices $V \in \mathbb{R}^{2 \times 2}$ and for all vectors $v \in \mathbb{R}^{2}$, and if the density function of $\zeta$ is positive on a set $S \subset \mathbb{R}^{2}$ then the density function of $V \zeta+v$ is positive on the set $V S+v$. Hence, it is enough to check that the random vector

$$
\left(\int_{0}^{1} \mathrm{e}^{b u} \sqrt{Y_{u}} \mathrm{~d} L_{u}, \int_{0}^{1} \mathrm{e}^{\theta u} \sqrt{Y_{u}} \mathrm{~d} B_{u}\right)
$$

is absolutely continuous with respect to the Lebesgue measure on $\mathbb{R}^{2}$ having a density function being strictly positive on the set $\left\{y \in \mathbb{R}: y>-y_{0}-a \int_{0}^{1} \mathrm{e}^{b u} \mathrm{~d} u\right\} \times \mathbb{R}$. For all $x \in \mathbb{R}$ and $y \leq-y_{0}-a \int_{0}^{1} \mathrm{e}^{b u} \mathrm{~d} u$, we have

$$
\begin{aligned}
& \mathbb{P}\left(\int_{0}^{1} \mathrm{e}^{b u} \sqrt{Y_{u}} \mathrm{~d} L_{u}<y, \int_{0}^{1} \mathrm{e}^{\theta u} \sqrt{Y_{u}} \mathrm{~d} B_{u}<x\right) \\
& \quad=\mathbb{P}\left(\mathrm{e}^{b} Y_{1}-y_{0}-a \int_{0}^{1} \mathrm{e}^{b u} \mathrm{~d} u<y, \int_{0}^{1} \mathrm{e}^{\theta u} \sqrt{Y_{u}} \mathrm{~d} B_{u}<x\right) \\
& \quad \leq \mathbb{P}\left(Y_{1}<0\right) \\
& \quad=0
\end{aligned}
$$

since $\mathbb{P}\left(Y_{1} \geq 0\right)=1$. Note that the conditional distribution of $\int_{0}^{1} \mathrm{e}^{\theta u} \sqrt{Y_{u}} \mathrm{~d} B_{u}$ given $\left(Y_{t}\right)_{t \in[0,1]}$ is a normal distribution with mean zero and variance $\int_{0}^{1} \mathrm{e}^{2 \theta u} Y_{u} \mathrm{~d} u$ due to the fact 
that $\left(Y_{t}\right)_{t \in[0,1]}$ and $\left(B_{t}\right)_{t \geq 0}$ are independent. Indeed, $\left(Y_{t}\right)_{t \geq 0}$ is adapted to the augmented filtration corresponding to $\eta_{0}$ and $\left(L_{t}\right)_{t \geq 0}$ (see, e.g. [22, p. 285]), and using the independence of the standard Wiener processes $L$ and $B$, and Problem 2.7.3 of [22], we can argue that this augmented filtration is independent of the filtration generated by $B$. Hence, again using the independence of the standard Wiener processes $L$ and $B$, we obtain, for all $x \in \mathbb{R}$ and $y>-y_{0}-a \int_{0}^{1} \mathrm{e}^{b u} \mathrm{~d} u$,

$$
\begin{aligned}
\mathbb{P}\left(\int_{0}^{1} \mathrm{e}^{b u} \sqrt{Y_{u}} \mathrm{~d} L_{u}<y, \int_{0}^{1} \mathrm{e}^{\theta u} \sqrt{Y_{u}} \mathrm{~d} B_{u}<x\right) \\
\quad=\mathbb{P}\left(\mathrm{e}^{b} Y_{1}-y_{0}-a \int_{0}^{1} \mathrm{e}^{b u} \mathrm{~d} u<y, \int_{0}^{1} \mathrm{e}^{\theta u} \sqrt{Y_{u}} \mathrm{~d} B_{u}<x\right) \\
\quad=\mathbb{E}\left(\mathbf{1}_{\left\{Y_{1}<\mathrm{e}^{-b}\left(y+y_{0}+a \int_{0}^{1} \mathrm{e}^{b u} \mathrm{~d} u\right)\right\}} \mathbb{E}\left(\mathbf{1}_{\left\{\int_{0}^{1} \mathrm{e}^{\theta u} \sqrt{Y_{u}} \mathrm{~d} B_{u}<x\right\}} \mid\left(Y_{t}\right)_{t \in[0,1]}\right)\right) \\
\quad=\mathbb{E}\left(\mathbf{1}_{\left\{Y_{1}<\mathrm{e}^{-b}\left(y+y_{0}+a \int_{0}^{1} \mathrm{e}^{b u} \mathrm{~d} u\right)\right\}} \int_{-\infty}^{x} p\left(w ; \int_{0}^{1} \mathrm{e}^{2 \theta u} Y_{u} \mathrm{~d} u\right) \mathrm{d} w\right),
\end{aligned}
$$

where

$$
p\left(w ; \sigma^{2}\right):=\frac{1}{\sqrt{2 \pi \sigma^{2}}} \mathrm{e}^{-w^{2} / 2 \sigma^{2}}, \quad w \in \mathbb{R}, \quad \sigma>0 .
$$

We note that, under the assumption that $a>0, \mathbb{P}\left(\int_{0}^{1} \mathrm{e}^{2 \theta u} Y_{u} \mathrm{~d} u>0\right)=1$. Then, by the law of total expectation, for all $x \in \mathbb{R}$ and $y>-y_{0}-a \int_{0}^{1} \mathrm{e}^{b u} \mathrm{~d} u$,

$$
\begin{aligned}
& \mathbb{P}\left(\int_{0}^{1} \mathrm{e}^{b u} \sqrt{Y_{u}} \mathrm{~d} L_{u}<y, \int_{0}^{1} \mathrm{e}^{\theta u} \sqrt{Y_{u}} \mathrm{~d} B_{u}<x\right) \\
& \quad=\int_{0}^{F} \int_{-\infty}^{x} \mathbb{E}\left(\frac{1}{\sqrt{2 \pi \int_{0}^{1} \mathrm{e}^{2 \theta u} Y_{u} \mathrm{~d} u}} \exp \left\{-\frac{w^{2}}{2 \int_{0}^{1} \mathrm{e}^{2 \theta u} Y_{u} \mathrm{~d} u}\right\} \mid Y_{1}=z\right) f_{Y_{1}}(z) \mathrm{d} z \mathrm{~d} w,
\end{aligned}
$$

where $F:=\mathrm{e}^{-b}\left(y+y_{0}+a \int_{0}^{1} \mathrm{e}^{b u} \mathrm{~d} u\right)$ and $f_{Y_{1}}$ denotes the density function of $Y_{1}$ (given that $\left.Y_{0}=y_{0}, y_{0} \in \mathbb{R}_{+}\right)$. For $y_{0} \in(0, \infty)$,

$$
f_{Y_{1}}(y)=\frac{2 b \mathrm{e}^{b(2 a+1)}}{\mathrm{e}^{b}-1}\left(\frac{y}{y_{0}}\right)^{a-1 / 2} \exp \left\{-\frac{2 b\left(y_{0}+\mathrm{e}^{b} y\right)}{\mathrm{e}^{b}-1}\right\} I_{2 a-1}\left(\frac{2 b \sqrt{y_{0} y}}{\sinh (b / 2)}\right) \mathbf{1}_{(0, \infty)}(y)
$$

for $y \in \mathbb{R}$, where $I_{2 a-1}$ denotes the modified Bessel of the first kind of order $2 a-1$, i.e.

$$
I_{2 a-1}(x)=\sum_{m=0}^{\infty} \frac{1}{m ! \Gamma(m+2 a)}\left(\frac{x}{2}\right)^{2 m+2 a-1}, \quad x \in(0, \infty) ;
$$

see, e.g. [5, Proof of Proposition 2], [10, Equation (18)], or [20, Proposition 6.3.2.1]. For $y_{0}=0$, the density function of $Y_{1}$ (given that $Y_{0}=0$ ) is given by

$$
f_{Y_{1}}(y)=\frac{1}{\Gamma(2 a)}\left(\frac{2 b}{1-\mathrm{e}^{-b}}\right)^{2 a} y^{2 a-1} \exp \left\{-\frac{2 b y}{1-\mathrm{e}^{-b}}\right\} \mathbf{1}_{(0, \infty)}(y), \quad y \in \mathbb{R},
$$

since, by [19, p. 222], $Y_{1}$ (given that $Y_{0}=0$ ) has a gamma distribution with parameters $2 a$ and $2 b /\left(1-\mathrm{e}^{-b}\right)$. Note that in both cases $f_{Y_{1}}(y)>0$ for all $y \in(0, \infty)$. Then, by a change of 
variable, we have, for all $x \in \mathbb{R}$ and $y>-y_{0}-a \int_{0}^{1} \mathrm{e}^{b u} \mathrm{~d} u$,

$$
\begin{gathered}
\mathbb{P}\left(\int_{0}^{1} \mathrm{e}^{b u} \sqrt{Y_{u}} \mathrm{~d} L_{u}<y, \int_{0}^{1} \mathrm{e}^{\theta u} \sqrt{Y_{u}} \mathrm{~d} B_{u}<x\right) \\
=\int_{-y_{0}-a \int_{0}^{1} \mathrm{e}^{b u} \mathrm{~d} u}^{y} \int_{-\infty}^{x} \mathbb{E}\left(\frac{\exp \left\{-w^{2} / 2 \int_{0}^{1} \mathrm{e}^{2 \theta u} Y_{u} \mathrm{~d} u\right\}}{\sqrt{2 \pi \int_{0}^{1} \mathrm{e}^{2 \theta u} Y_{u} \mathrm{~d} u}} \mid Y_{1}=\mathrm{e}^{-b}\left(v+y_{0}+a \int_{0}^{1} \mathrm{e}^{b u} \mathrm{~d} u\right)\right) \\
\times f_{Y_{1}}\left(\mathrm{e}^{-b}\left(v+y_{0}+a \int_{0}^{1} \mathrm{e}^{b u} \mathrm{~d} u\right)\right) \mathrm{e}^{-b} \mathrm{~d} v \mathrm{~d} w .
\end{gathered}
$$

In what follows we will make use of the simple observation that, if $\xi$ and $\eta$ are random variables such that $\mathbb{P}(\xi>0)=1, \mathbb{E}(\xi)<\infty$, and $\mathbb{P}(\eta>0)=1$, and $\eta$ is absolutely continuous with a density function $f_{\eta}$ having the property $f_{\eta}(x)>0$ Lebesgue almost every $x \in(0, \infty)$, then $\mathbb{E}(\xi \mid \eta=y)>0$ Lebesgue almost every $y \in(0, \infty)$. For a proof, see, e.g. the proof of Theorem 4.1 of [3].

Now we return to the proof that the random vector (4.3) is absolutely continuous with respect to the Lebesgue measure on $\mathbb{R}^{2}$ with a density function being strictly positive on the set $\left\{y \in \mathbb{R}: y>-y_{0}-a \int_{0}^{1} \mathrm{e}^{b u} \mathrm{~d} u\right\} \times \mathbb{R}$. Since $\sup _{x \in \mathbb{R}_{+}} x \mathrm{e}^{-w^{2} x^{2} / 2}<\infty, w \in \mathbb{R} \backslash\{0\}$, implies that

$$
\mathbb{E}\left(\left(2 \pi \int_{0}^{1} \mathrm{e}^{2 \theta u} Y_{u} \mathrm{~d} u\right)^{1 / 2} \exp \left\{-\frac{w^{2}}{2 \int_{0}^{1} \mathrm{e}^{2 \theta u} Y_{u} \mathrm{~d} u}\right\}\right)<\infty, \quad w \in \mathbb{R} \backslash\{0\},
$$

and using the fact that $f_{Y_{1}}\left(\mathrm{e}^{-b}\left(v+y_{0}+a \int_{0}^{1} \mathrm{e}^{b u} \mathrm{~d} u\right)\right)>0$ for all $v>-y_{0}-a \int_{0}^{1} \mathrm{e}^{b u} \mathrm{~d} u$, there exists a measurable function $g: \mathbb{R}^{2} \rightarrow \mathbb{R}_{+}$such that $g(v, w)>0$ for $v>-y_{0}-$ $a \int_{0}^{1} \mathrm{e}^{b u} \mathrm{~d} u, w \in \mathbb{R}$, and

$$
\begin{aligned}
& \mathbb{P}\left(\int_{0}^{1} \mathrm{e}^{b u} \sqrt{Y_{u}} \mathrm{~d} L_{u}<y, \int_{0}^{1} \mathrm{e}^{\theta u} \sqrt{Y_{u}} \mathrm{~d} B_{u}<x\right) \\
& \quad= \begin{cases}\int_{-y_{0}-a \int_{0}^{1} \mathrm{e}^{b u} \mathrm{~d} u}^{y} \int_{-\infty}^{x} g(v, w) \mathrm{d} v \mathrm{~d} w & \text { if } y>-y_{0}-a \int_{0}^{1} \mathrm{e}^{b u} \mathrm{~d} u, x \in \mathbb{R}, \\
0 & \text { if } y \leq-y_{0}-a \int_{0}^{1} \mathrm{e}^{b u} \mathrm{~d} u, x \in \mathbb{R},\end{cases}
\end{aligned}
$$

as desired. Consequently, the random vector (4.3) is absolutely continuous with density function $g$ having the desired property.

To prove (c), we first note that, since the sample paths of $(Y, X)$ are almost surely continuous for each $n \in \mathbb{N}$, the extended generator has the form

$$
\left(\mathcal{A}_{n} f\right)(y, x)=\frac{1}{2} y f_{1,1}^{\prime \prime}(y, x)+\frac{1}{2} y f_{2,2}^{\prime \prime}(y, x)+(a-b y) f_{1}^{\prime}(y, x)+(m-\theta x) f_{1}^{\prime}(y, x)
$$

for all $(y, x) \in O_{n}$ and $f \in \mathcal{C}^{2}\left(\mathbb{R}_{+} \times \mathbb{R}, \mathbb{R}\right)$; see, e.g. [29, p. 538]. We also note that, by [13, Theorem 2.7], for functions $f \in \mathcal{C}_{\mathrm{c}}^{2}\left(\mathbb{R}_{+} \times \mathbb{R}, \mathbb{R}\right), \mathcal{A}_{n} f=\mathcal{A} f$ on $O_{n}$, where $\mathcal{A}$ denotes the (nonextended) generator of the process $\left(Y_{t}, X_{t}\right)_{t \in \mathbb{R}_{+}}$. For the function $V$ defined in (4.2), we have $V \in \mathcal{C}^{2}\left(\mathbb{R}_{+} \times \mathbb{R}, \mathbb{R}\right)$ and

$$
V_{1}^{\prime}(y, x)=2\left(y-c_{1}\right), \quad V_{2}^{\prime}(y, x)=2\left(x-c_{1}\right), \quad V_{1,1}^{\prime \prime}(y, x)=V_{2,2}^{\prime \prime}(y, x)=2,
$$

for $(y, x) \in \mathbb{R}_{+} \times \mathbb{R}$, and, hence, for all $n \in \mathbb{N}$ and $0<c<\infty$,

$$
\begin{aligned}
\left(\mathcal{A}_{n} V\right)(y, x)+c V(y, x)= & (c-2 b) y^{2}+2\left(1+a+b c_{1}-c c_{1}\right) y+c c_{1}^{2}-2 a c_{1} \\
& +2(m-\theta x)\left(x-c_{2}\right)+c\left(x-c_{2}\right)^{2} \text { for all }(y, x) \in O_{n} .
\end{aligned}
$$


Let us choose $c>0$ in such a way that $c \neq 2 b$, and let $c_{2}:=m / \theta$. Then

$$
\begin{aligned}
\left(\mathscr{A}_{n} V\right)(y, x)+c V(y, x)= & (c-2 b)\left(y+\frac{1+a+b c_{1}-c c_{1}}{c-2 b}\right)^{2}-\frac{\left(1+a+b c_{1}-c c_{1}\right)^{2}}{c-2 b} \\
& +c c_{1}^{2}-2 a c_{1}+(c-2 \theta)\left(x-\frac{m}{\theta}\right)^{2}, \quad(y, x) \in O_{n} .
\end{aligned}
$$

Hence, $\left(\mathcal{A}_{n} V\right)(y, x) \leq-c V(y, x)+d,(y, x) \in O_{n}, n \in \mathbb{N}$, with $c \in(0,2 \min (b, \theta))$ and

$$
d:=-\frac{\left(1+a+b c_{1}-c c_{1}\right)^{2}}{c-2 b}+c c_{1}^{2}-2 a c_{1} .
$$

Note that the above argument also shows that in the definition of the function $V$ given in (4.2) the constant $c_{1}$ can be an arbitrary real number.

In the next theorem we collect several facts about the random variable $\left(Y_{\infty}, X_{\infty}\right)$ given by (3.1) and (3.2) with $\alpha=2$.

Theorem 4.2. The random variable $\left(Y_{\infty}, X_{\infty}\right)$ given by (3.1) and (3.2) with $\alpha=2$ is absolutely continuous, and the Laplace transform of $Y_{\infty}$ takes the form

$$
\mathbb{E}\left(\mathrm{e}^{-\lambda_{1} Y_{\infty}}\right)=\left(1+\frac{\lambda_{1}}{2 b}\right)^{-2 a}, \quad \lambda_{1} \in \mathbb{R}_{+},
$$

so $Y_{\infty}$ has gamma distribution with parameters $2 a$ and $2 b$. Furthermore, all the (mixed) moments of $\left(Y_{\infty}, X_{\infty}\right)$ of any order are finite, i.e. we have $\mathbb{E}\left(Y_{\infty}^{n}\left|X_{\infty}\right|^{p}\right)<\infty$ for all $n, p \in \mathbb{Z}_{+}$, and, specifically,

$$
\begin{gathered}
\mathbb{E}\left(Y_{\infty}\right)=\frac{a}{b}, \quad \mathbb{E}\left(X_{\infty}\right)=\frac{m}{\theta}, \\
\mathbb{E}\left(Y_{\infty}^{2}\right)=\frac{a(2 a+1)}{2 b^{2}}, \quad \mathbb{E}\left(Y_{\infty} X_{\infty}\right)=\frac{m a}{\theta b}, \quad \mathbb{E}\left(X_{\infty}^{2}\right)=\frac{a \theta+2 b m^{2}}{2 b \theta^{2}}, \\
\mathbb{E}\left(Y_{\infty} X_{\infty}^{2}\right)=\frac{a}{(b+2 \theta) 2 b^{2} \theta^{2}}\left(\theta(a b+2 a \theta+\theta)+2 m^{2} b(2 \theta+b)\right) .
\end{gathered}
$$

Proof. First we show that the random variable $\left(Y_{\infty}, X_{\infty}\right)$ is absolutely continuous. Let us consider the two-dimensional affine diffusion model (1.1) with $\alpha=2, a>0, b>0, m \in \mathbb{R}$, and $\theta>0$, and with a random initial value $\left(Y_{0}, X_{0}\right)$ independent of $\left(L_{t}, B_{t}\right)_{t \geq 0}$ having the same distribution as that of $\left(Y_{\infty}, X_{\infty}\right)$. Then, by part (ii) of Theorem 3.1, the process $\left(Y_{t}, X_{t}\right)_{t \geq 0}$ is strictly stationary. Hence, it is enough to prove that $\left(Y_{1}, X_{1}\right)$ is absolutely continuous. This can be done similarly to the proof of Theorem 4.1(b), and so we only sketch a proof. Namely, by Theorem 2.1 we have

$$
\begin{aligned}
& Y_{1}=\mathrm{e}^{-b}\left(Y_{0}+a \int_{0}^{1} \mathrm{e}^{b u} \mathrm{~d} u+\int_{0}^{1} \mathrm{e}^{b u} \sqrt{Y_{u}} \mathrm{~d} L_{u}\right), \\
& X_{1}=\mathrm{e}^{-\theta}\left(X_{0}+m \int_{0}^{1} \mathrm{e}^{\theta u} \mathrm{~d} u+\int_{0}^{1} \mathrm{e}^{\theta u} \sqrt{Y_{u}} \mathrm{~d} B_{u}\right) .
\end{aligned}
$$

Since $\xi$ and $\eta$ are independent two-dimensional random vectors such that one is absolutely continuous, their sum $\xi+\eta$ is absolutely continuous (regardless of the other distribution), so it is enough to check that the random vector

$$
\left(\int_{0}^{1} \mathrm{e}^{b u} \sqrt{Y_{u}} \mathrm{~d} L_{u}, \int_{0}^{1} \mathrm{e}^{\theta u} \sqrt{Y_{u}} \mathrm{~d} B_{u}\right)
$$


is absolutely continuous with respect to the Lebesgue measure on $\mathbb{R}^{2}$. Its proof goes along the very same lines as the proof of Theorem 4.1(b).

The fact that $Y_{\infty}$ has a gamma distribution with parameters $2 a$ and $2 b$ follows from [10, Equation (20)]. For other proofs, see [3, Proof of Theorem 4.2].

We now prove that all the mixed moments of $\left(Y_{\infty}, X_{\infty}\right)$ are finite. Let us consider the two-dimensional affine diffusion model (1.1) with $\alpha=2, a>0, b>0, m \in \mathbb{R}$, and $\theta>0$, and with a random initial value $\left(Y_{0}, X_{0}\right)$ independent of $\left(L_{t}, B_{t}\right)_{t \geq 0}$ such that all the mixed moments of $\left(Y_{0}, X_{0}\right)$ are finite and $\mathbb{P}\left(Y_{0}>0\right)=1$. We note that, due to Theorem 3.1, the distribution of $\left(Y_{\infty}, X_{\infty}\right)$ does not depend on the initial value of model (1.1), so we can have such a choice. First we show that

$$
\int_{0}^{t} \mathbb{E}\left(Y_{u}^{n} X_{u}^{2 p}\right) \mathrm{d} u<\infty \quad \text { for all } t \geq 0 \text { and } n, p \in \mathbb{Z}_{+} .
$$

Owing to the inequality between two power means, $(a+b+c)^{2 p} \leq K\left(a^{2 p}+b^{2 p}+\right.$ $\left.c^{2 p}\right), a, b, c \in \mathbb{R}$, with some constant $K>0$, and, hence, by (2.2), to prove (4.4), it is enough to check that

$$
\begin{array}{rl}
\int_{0}^{t} & \mathbb{E}\left(Y_{u}^{n}\left(\mathrm{e}^{-2 p \theta u} X_{0}^{2 p}+\left(m \int_{0}^{u} \mathrm{e}^{-\theta(u-v)} \mathrm{d} v\right)^{2 p}+\left(\int_{0}^{u} \mathrm{e}^{-\theta(u-v)} \sqrt{Y_{u}} \mathrm{~d} B_{v}\right)^{2 p}\right)\right) \mathrm{d} u \\
<\infty &
\end{array}
$$

for all $t \geq 0$ and $n, p \in \mathbb{Z}_{+}$. Since, for all $u \in[0, t]$, the conditional distribution of $\int_{0}^{u} \mathrm{e}^{-\theta(u-\bar{v})} \sqrt{Y_{v}} \mathrm{~d} B_{v}$ with respect to the $\sigma$-algebra generated by $\left(Y_{s}\right)_{s \in[0, t]}$ is a normal distribution with mean 0 and variance $\int_{0}^{u} \mathrm{e}^{-2 \theta(u-v)} Y_{v} \mathrm{~d} v$, to prove (4.5), it is enough to check that, for all $t \geq 0$ and $n, p \in \mathbb{Z}_{+}$,

$$
\int_{0}^{t} \mathbb{E}\left(\mathrm{e}^{-2 p \theta u} Y_{u}^{n} X_{0}^{2 p}\right) \mathrm{d} u<\infty, \quad \int_{0}^{t} \mathbb{E}\left(Y_{u}^{n}\right) \mathrm{d} u<\infty,
$$

and

$$
\int_{0}^{t} \mathbb{E}\left(Y_{u}^{n}\left(\int_{0}^{u} \mathrm{e}^{-2 \theta(u-v)} Y_{v} \mathrm{~d} v\right)^{p}\right) \mathrm{d} u<\infty .
$$

Using the facts that $\theta>0$ and $\mathbb{P}\left(Y_{t} \geq 0, t \in \mathbb{R}_{+}\right)=1$, by the Cauchy-Schwarz inequality, for all $u \in[0, t]$, we have $\mathbb{E}\left(\mathrm{e}^{-2 p \theta u} Y_{u}^{n} X_{0}^{2 p}\right) \leq\left(\mathbb{E}\left(Y_{u}^{2 n}\right)\right)^{1 / 2}\left(\mathbb{E}\left(X_{0}^{4 p}\right)\right)^{1 / 2}$, and

$$
\begin{aligned}
\mathbb{E}\left(Y_{u}^{n}\right. & \left.\left(\int_{0}^{u} \mathrm{e}^{-2 \theta(u-v)} Y_{v} \mathrm{~d} v\right)^{p}\right) \\
& \leq\left(\mathbb{E}\left(Y_{u}^{2 n}\right)\right)^{1 / 2}\left(\mathbb{E}\left(\left(\int_{0}^{u} Y_{v} \mathrm{~d} v\right)^{2 p}\right)\right)^{1 / 2} \\
& =\left(\mathbb{E}\left(Y_{u}^{2 n}\right)\right)^{1 / 2}\left(\int_{0}^{u} \cdots \int_{0}^{u} \mathbb{E}\left(Y_{v_{1}} \cdots Y_{v_{2 p}}\right) \mathrm{d} v_{1} \cdots \mathrm{d} v_{2 p}\right)^{1 / 2} \\
& \leq\left(\mathbb{E}\left(Y_{u}^{2 n}\right)\right)^{1 / 2}\left(\int_{0}^{u} \cdots \int_{0}^{u}\left(\mathbb{E}\left(Y_{v_{1}}^{2 p}\right) \cdots \mathbb{E}\left(Y_{v_{2} p}^{2 p}\right)\right)^{1 / 2 p} \mathrm{~d} v_{1} \cdots \mathrm{d} v_{2 p}\right)^{1 / 2},
\end{aligned}
$$

where the last inequality follows by the multivariate version of Hölder's inequality. Since $\mathbb{E}\left(X_{0}^{4 p}\right)<\infty$, this shows that, in order to prove (4.4), it is enough to check that, for all $k \in \mathbb{Z}_{+}$ and $t \in \mathbb{R}_{+}$, $\sup _{u \in[0, t]} \mathbb{E}\left(Y_{u}^{k}\right)<\infty$, which is verified in [3, Proof of Theorem 4.2]. 
For all $n, p \in \mathbb{Z}_{+}$, using the independence of $L$ and $B$, by Itô's formula, we have

$$
\begin{aligned}
\mathrm{d}\left(Y_{t}^{n} X_{t}^{p}\right)= & n Y_{t}^{n-1} X_{t}^{p}\left(\left(a-b Y_{t}\right) \mathrm{d} t+\sqrt{Y_{t}} \mathrm{~d} L_{t}\right)+p Y_{t}^{n} X_{t}^{p-1}\left(\left(m-\theta X_{t}\right) \mathrm{d} t+\sqrt{Y_{t}} \mathrm{~d} B_{t}\right) \\
& +\frac{n(n-1)}{2} Y_{t}^{n-2} X_{t}^{p} Y_{t} \mathrm{~d} t+\frac{p(p-1)}{2} Y_{t}^{n} X_{t}^{p-2} Y_{t} \mathrm{~d} t \\
= & \left(n Y_{t}^{n-1}\left(a-b Y_{t}\right) X_{t}^{p}+p Y_{t}^{n}\left(m-\theta X_{t}\right) X_{t}^{p-1}+\frac{n(n-1)}{2} Y_{t}^{n-1} X_{t}^{p}\right. \\
& \left.+\frac{p(p-1)}{2} Y_{t}^{n+1} X_{t}^{p-2}\right) \mathrm{d} t+n Y_{t}^{n-1 / 2} X_{t}^{p} \mathrm{~d} L_{t}+p Y_{t}^{n+1 / 2} X_{t}^{p-1} \mathrm{~d} B_{t}
\end{aligned}
$$

for $t \geq 0$. Writing the SDE above in an integrated form and taking the expectation of both sides, we have

$$
\begin{aligned}
& \mathbb{E}\left(Y_{t}^{n} X_{t}^{p}\right)-\mathbb{E}\left(Y_{0}^{n} X_{0}^{p}\right) \\
& =\int_{0}^{t}\left[\text { an } \mathbb{E}\left(Y_{u}^{n-1} X_{u}^{p}\right)-b n \mathbb{E}\left(Y_{u}^{n} X_{u}^{p}\right)+p m \mathbb{E}\left(Y_{u}^{n} X_{u}^{p-1}\right)-p \theta \mathbb{E}\left(Y_{u}^{n} X_{u}^{p}\right)\right. \\
& \left.\quad+\frac{n(n-1)}{2} \mathbb{E}\left(Y_{u}^{n-1} X_{u}^{p}\right)+\frac{p(p-1)}{2} \mathbb{E}\left(Y_{u}^{n+1} X_{u}^{p-2}\right)\right] \mathrm{d} u, \quad t \geq 0,
\end{aligned}
$$

where we have used the facts that

$$
\left(\int_{0}^{t} Y_{u}^{n-1 / 2} X_{u}^{p} \mathrm{~d} L_{u}\right)_{t \geq 0} \text { and }\left(\int_{0}^{t} Y_{u}^{n+1 / 2} X_{u}^{p-1} \mathrm{~d} B_{u}\right)_{t \geq 0}
$$

are continuous square-integrable martingales due to (4.4); see, e.g. [19, p. 55]. Introduce the functions $f_{n, p}(t):=\mathbb{E}\left(Y_{t}^{n} X_{t}^{p}\right), t \in \mathbb{R}_{+}$, for $n, p \in \mathbb{Z}_{+}$. Then we have

$$
\begin{aligned}
f_{n, p}^{\prime}(t)= & -(b n+p \theta) f_{n, p}(t)+\left(a n+\frac{n(n-1)}{2}\right) f_{n-1, p}(t)+p m f_{n, p-1}(t) \\
& +\frac{p(p-1)}{2} f_{n+1, p-2}(t), \quad t \in \mathbb{R}_{+},
\end{aligned}
$$

where $f_{k, \ell}(t):=0$ if $k, \ell \in \mathbb{Z}$ with $k<0$ or $\ell<0$. Hence, for all $M \in \mathbb{N}$, the functions $f_{n, p}, n, p \in \mathbb{Z}_{+}$, with $n+p \leq M$ satisfy a homogeneous linear system of differential equations with constant coefficients. Thus, for all $n, p \in \mathbb{Z}_{+}$, the function $f_{n, p}$ is a linear combination of the functions $\mathrm{e}^{-(k b+\ell \theta) t}, t \in \mathbb{R}_{+}, k, \ell \in \mathbb{Z}_{+}$, with $k+\ell \leq n+p$, since the eigenvalues of the coefficient matrix of the abovementioned system of differential equations are $-(k b+\ell \theta), k, \ell \in$ $\mathbb{Z}_{+}$, with $k+\ell \leq M$. Consequently, for all $n, p \in \mathbb{Z}_{+}$, the function $f_{n, p}$ is bounded and the $\operatorname{limit}_{t \rightarrow \infty} \lim _{n, p}(t)$ exists and is finite. By the moment convergence theorem (see, e.g. [33, Lemma 2.2.1]), $\lim _{t \rightarrow \infty} f_{n, p}(t)=\lim _{t \rightarrow \infty} \mathbb{E}\left(Y_{t}^{n} X_{t}^{p}\right)=\mathbb{E}\left(Y_{\infty}^{n} X_{\infty}^{p}\right), n, p \in \mathbb{Z}_{+}$. Indeed, by Theorem 3.1 and the continuous mapping theorem, $Y_{t}^{n} X_{t}^{p} \stackrel{\mathrm{D}}{\rightarrow} Y_{\infty}^{n} X_{\infty}^{p}$ as $t \rightarrow \infty$, and the family $\left\{Y_{t}^{n} X_{t}^{p}: t \in \mathbb{R}_{+}\right\}$is uniformly integrable. This latter fact follows from the boundedness of the function $f_{2 n, 2 p}$; see, e.g. [33, Condition (2.2.5)]. Hence, all the mixed moments of $\left(Y_{\infty}, X_{\infty}\right)$ are finite.

Finally, we calculate the moments listed in the theorem. Let us consider again the twodimensional affine diffusion model (1.1) with $\alpha=2, a>0, b>0, m \in \mathbb{R}$, and $\theta>0$, and with a random initial value $\left(Y_{0}, X_{0}\right)$ independent of $\left(L_{t}, B_{t}\right)_{t \geq 0}$ having the same distribution as that of $\left(Y_{\infty}, X_{\infty}\right)$. Then, by Theorem 3.1, the process $\left(Y_{t}, X_{t}\right)_{t \geq 0}$ is strictly stationary, and, 
hence, $f_{n, p}(t)=\mathbb{E}\left(Y_{\infty}^{n} X_{\infty}^{p}\right)$ for all $t \in \mathbb{R}_{+}$and $n, p \in \mathbb{Z}_{+}$. The above system of differential equations for the functions $f_{n, p}, n, p \in \mathbb{Z}_{+}$, yields

$$
\begin{aligned}
\mathbb{E}\left(Y_{\infty}^{n} X_{\infty}^{p}\right)=\frac{1}{b n+p \theta}( & \left(a n+\frac{n(n-1)}{2}\right) \mathbb{E}\left(Y_{\infty}^{n-1} X_{\infty}^{p}\right)+m p \mathbb{E}\left(Y_{\infty}^{n} X_{\infty}^{p-1}\right) \\
& \left.+\frac{p(p-1)}{2} \mathbb{E}\left(Y_{\infty}^{n+1} X_{\infty}^{p-2}\right)\right)
\end{aligned}
$$

for all $n, p \in \mathbb{Z}_{+}$. By (4.6) we can calculate the moments listed in the theorem.

Finally, we note that, to calculate the moments $\mathbb{E}\left(Y_{\infty}^{n} X_{\infty}^{p}\right)<\infty, n, p \in \mathbb{Z}_{+}$, we could have used Formula (4.4) of [15], which gives a formal representation of the polynomial moments of $\left(Y_{t}, X_{t}\right), t \in \mathbb{R}_{+}$. The idea behind this formal representation is that the infinitesimal generator of the affine process $(Y, X)$ formally maps the finite-dimensional linear space of all polynomials in $(y, x) \in \mathbb{R}_{+} \times \mathbb{R}$ of degree less than or equal to $k$ into itself, where $k \in \mathbb{N}$. For a more general class of time-homogeneous Markov processes having this property, for the so-called polynomial processes, see [11]. We also remark that the moments of $Y_{\infty}$ could have been calculated directly using the fact that $Y_{\infty}$ has a gamma distribution with parameters $2 a$ and $2 b$.

\section{Acknowledgements}

We are undoubtedly grateful to the anonymous referee for pointing out a mistake in the proof of Theorem 4.1, and also for his/her several valuable comments that have led to an improvement of the manuscript. The research of M. Barczy and G. Pap was realized in the frames of TÁMOP 4.2.4. A/2-11-1-2012-0001, 'National Excellence Program - Elaborating and operating an inland student and researcher personal support system'. The project was subsidized by the European Union and co-financed by the European Social Fund. Z. Li was partially supported by the NSFC under grant number 11131003 and the 973 Program under grant number 2011CB808001.

\section{References}

[1] Andersen, L. B. G. And Piterbarg, V. V. (2007). Moment explosions in stochastic volatility models. Finance Stoch. 11, 29-50.

[2] Barczy, M., Döring, L., Li, Z. ANd PAP, G. (2013). On parameter estimation for critical affine processes. Electron. J. Statist. 7, 647-696.

[3] Barczy, M., DöRIng, L., Li, Z. AND Pap, G. (2013). Stationarity and ergodicity for an affine two factor model. Preprint. Available at http://uk.arxiv.org/abs/1302.2534v4.

[4] Barndorff-Nielsen, O. And Shephard, N. (2001). Non-Gaussian Ornstein-Uhlenbeck-based models and some of their uses in financial economics. J. R. Statist. Soc. B 63, 167-241.

[5] Ben Alaya, M. and Kebaier, A. (2013). Asymptotic behavior of the maximum likelihood estimator for ergodic and nonergodic square-root diffusions. Stoch. Anal. Appl. 31, 552-573.

[6] Bhattacharya, R. N. (1982). On the functional central limit theorem and the law of the iterated logarithm for Markov processes. Z. Wahrscheinlichkeitsth. 60, 185-201.

[7] CARr, P. AND Wu, L. (2003). The finite moment log stable process and option pricing. J. Finance 58, 753-777.

[8] Chen, H. And Joslin, S. (2012). Generalized transform analysis of affine processes and applications in finance. Rev. Financial Stud. 25, 2225-2256.

[9] Chung, K. L. (1982). Lectures from Markov Processes to Brownian Motion. Springer, New York.

[10] Cox, J. C., Ingersoll, J. E., JR. And Ross, S. A. (1985). A theory of the term structure of interest rates. Econometrica 53, 385-407.

[11] Cuchiero, C., Keller-Ressel, M. and Teichmann, J. (2012). Polynomial processes and their applications to mathematical finance. Finance Stoch. 16, 711-740.

[12] Dawson, D. A. And Li, Z. (2006). Skew convolution semigroups and affine Markov processes. Ann. Prob. 34, 1103-1142. 
[13] Duffie, D., Filipović, D. And Schachermayer, W. (2003). Affine processes and applications in finance. Ann. Appl. Prob. 13, 984-1053.

[14] Filipović, D. and Mayerhofer, E. (2009). Affine diffusion processes: theory and applications. In Advanced Financial Modelling (Radon Ser. Comput. Appl. Math. 8), De Gruyter, Berlin, pp. 125-164.

[15] Filipović, D., Mayerhofer, E. And Schneider, P. (2013). Density approximations for multivariate affine jump-diffusion processes. J. Econometrics 176, 93-111.

[16] Fu, Z. AND Li, Z. (2010). Stochastic equations of non-negative processes with jumps. Stoch. Process. Appl. 120, 306-330.

[17] Glasserman, P. and Kim, K.-K. (2010). Moment explosions and stationary distributions in affine diffusion models. Math. Finance 20, 1-33.

[18] Heston, S. L. (1993). A closed-form solution for options with stochastic volatility with applications to bond and currency options. Rev. Financial Stud. 6, 327-343.

[19] IKeda, N. And Watanabe, S. (1981). Stochastic Differential Equations and Diffusion Processes. North-Holland, Amsterdam.

[20] Jeanblanc, M., Yor, M. and Chesney, M. (2009). Mathematical Methods for Financial Markets. Springer, London.

[21] Jena, R. P., Kim, K.-K. AND Xing, H. (2012). Long-term and blow-up behaviors of exponential moments in multi-dimensional affine diffusions. Stoch. Process. Appl. 122, 2961-2993.

[22] Karatzas, I. And Shreve, S. E. (1991). Brownian Motion and Stochastic Calculus, 2nd edn. Springer, New York.

[23] Keller-Ressel, M. and Mijatović, A. (2012). On the limit distributions of continuous-state branching processes with immigration. Stoch. Process. Appl. 122, 2329-2345.

[24] Keller-Ressel, M., Schachermayer, W. and Teichmann, J. (2011). Affine processes are regular. Prob. Theory Relat. Fields 151, 591-611.

[25] LI, Z. (2011). Measure-Valued Branching Markov Processes. Springer, Heidelberg.

[26] LI, Z. AND MA, C. (2013). Asymptotic properties of estimators in a stable Cox-Ingersoll-Ross model. Preprint. Available at http://uk.arxiv.org/abs/1301.3243.

[27] Meyn, S. P. AND Tweedie, R. L. (1992). Stability of Markovian processes. I. Criteria for discrete-time chains. Adv. Appl. Prob. 24, 542-574.

[28] Meyn, S. P. And Tweedie, R. L. (1993). Stability of Markovian processes. II. Continuous-time processes and sampled chains. Adv. Appl. Prob. 25, 487-517.

[29] Meyn, S. P. And Tweedie, R. L. (1993). Stability of Markovian processes. III. Foster-Lyapunov criteria for continuous-time processes. Adv. Appl. Prob. 25, 518-548.

[30] Meyn, S. P. AND Tweedie, R. L. (2009). Markov Chains and Stochastic Stability, 2nd edn. Cambridge University Press.

[31] Sandrić, N. (2013). Long-time behavior of stable-like processes. Stoch. Process. Appl. 123, 1276-1300.

[32] Sharpe, M. (1988). General Theory of Markov Processes. Academic Press, Boston, MA.

[33] Stroock, D. W. (1993). Probability Theory, An Analytic View. Cambridge University Press.

[34] Volkmann, P. (1972). Gewöhnliche Differentialungleichungen mit quasimonoton wachsenden funktionen in topologischen Vektorräumen. Math. Z. 127, 157-164.

[35] Zolotarev, V. M. (1986). One-Dimensional Stable Distributions (Transl. Math. Monogr. 65). American Mathematical Society, Providence, RI. 